\title{
Analysis of the hydrostatic Stokes problem and finite-element approximation in unstructured meshes*
}

\author{
F. Guillén-González ${ }^{\dagger}$ and J.R. Rodríguez-Galván
}

September 10, 2014

\begin{abstract}
The stability of velocity and pressure mixed finite-element approximations in general meshes of the hydrostatic Stokes problem is studied, where two "inf-sup" conditions appear associated to the two constraints of the problem; namely incompressibility and hydrostatic pressure. Since these two constraints have different properties, it is not easy to choose finite element spaces satisfying both. From the analytical point of view, two main results are established; the stability of an anisotropic approximation of the velocity (using different spaces for horizontal and vertical velocities) with piecewise constant pressures, and the unstability of standard (isotropic) approximations which are stable for the Stokes problem, like the mini-element or the Taylor-Hood element. Moreover, we give some numerical simulations, which agree with the previous analytical results and allow us to conjecture the stability of some anisotropic approximations of the velocity with continuous piecewise linear pressure in unstructured meshes.
\end{abstract}

Keywords. Inf-sup condition, incompressible fluids, hydrostatic pressure, primitive equations, finite elements, unstructured meshes, anisotropic Stokes equations.

AMS classification (2010). 35Q35, 65N12, 65N30, 76D07.

\section{Contents}

1 Introduction 2

2 Preliminaries and problem setting 4

3 Well-posedness of the hydrostatic Stokes problem: a first proof $\quad 7$

4 A new proof using the saddle point framework 11

${ }^{*}$ The first author has been partially supported by projects MTM2009-12927 and MTM2012-32325 (DGIMEC, Spain) and the second one by the research group FQM-315 (Junta de Andalucía).

${ }^{\dagger}$ Departamento EDAN and IMUS. Universidad de Sevilla. Aptdo. 1160, 41080 Sevilla (Spain). guillen@us.es

${ }^{\ddagger}$ Departamento de Matemáticas. Universidad de Cádiz. Polígono Río San Pedro S/N, 11510 Puerto Real, Cádiz (Spain). rafael.rodriguez@uca.es 
5 Conforming finite elements 13

5.1 Necessary and sufficient conditions for stability . . . . . . . . . . . . 13

5.2 Sufficient conditions for "incompressible" stability . . . . . . . . . . 14

5.3 Necessary conditions for stability . . . . . . . . . . . . . . . . 14

6 Applications and first numerical results $\quad 15$

6.1 Unstable approximations of the Stokes problem with vanishing vertical vis-

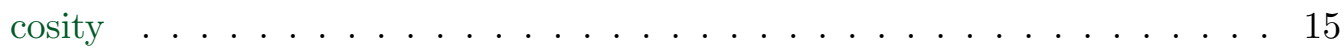

6.2 Unstable approximations of the hydrostatic Stokes problem . . . . . . . . 18

6.3 Stability of $\left(\mathcal{P}_{2}, \mathcal{P}_{1}\right)-\mathcal{P}_{0}$ for the hydrostatic Stokes problem . . . . . . . . 20

6.4 About the stability of $\left(\mathcal{P}_{1, b}, \mathcal{P}_{1}\right)-\mathcal{P}_{1}$ and $\left(\mathcal{P}_{2}, \mathcal{P}_{1}\right)-\mathcal{P}_{1}$ for the hydrostatic

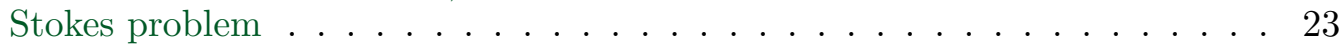

7 Numerical simulations with different domains $\quad 27$

\section{Introduction}

The equations of geophysical fluid dynamics, modeling the motion of the ocean and the atmosphere, are derived from the conservation laws from physics. In the case of large scale ocean, it is considered as made up of a slightly compressible fluid endowed with Coriolis force and the following set of laws (see e.g. [CB09, LTW92a, LTW92b, Ped87, Tem03, TZ04]): conservation of momentum, conservation of mass (continuity equation), thermodynamics equation (conservation of energy), convection-diffusion equation for the salinity and a state equation for the density (dependent on temperature and salinity).

The resulting system is too complicated for the purpose of fluid dynamics and, from a practical point of view, unaffordable. In consequence, some simplifications must be introduced. In this paper (see section 2) Cartesian coordinates in a shallow domain with the "rigid-lid" hypothesis (flat surface) is assumed. Moreover, for simplicity constant density (independent on temperature and salinity effects) is also assumed, although the results of this paper can be extended to a density-dependent case under the Boussinesq approximation. Finally, if the vertical momentum equation is reduced to the hydrostatic balance $\partial_{z} p=0$ (including the linear in depth hydrostatic pressure in the potential $p$ ), the well known primitive (or hydrostatic Navier-Stokes) equations (see (11)-(13) in Section 2) can be derived, in an oceanic domain $\Omega \subset \mathbb{R}^{3}$.

This paper focus on the theoretical and numerical study of the linear steady case, arriving at the so-called hydrostatic Stokes equations in $\Omega$ :

$$
\begin{aligned}
-\Delta_{\nu} \mathbf{u}+\nabla_{\mathbf{x}} p & =\mathbf{f}, \\
\partial_{z} p & =0, \\
\nabla \cdot(\mathbf{u}, v) & =0,
\end{aligned}
$$

enclosed with the boundary conditions given in (8)-(9). Here $(\mathbf{u}, v): \Omega \longrightarrow \mathbb{R}^{3}$ is the velocity field $\left(\mathbf{u}=\left(u_{1}, u_{2}\right)^{t}\right), p: \Omega \longrightarrow \mathbb{R}$ is the potential (depending on the pressure), and $\mathbf{f}=\left(f_{1}, f_{2}\right)^{t}$ is an external force. We are denoting $\nabla_{\mathbf{x}}=\left(\partial_{x}, \partial_{y}\right)^{t}, \nabla_{\mathbf{x}} \cdot \mathbf{u}=\partial_{x} u_{1}+\partial_{y} u_{2}$, $\nabla \cdot(\mathbf{u}, v)=\nabla_{\mathbf{x}} \cdot \mathbf{u}+\partial_{z} v$ and $\Delta_{\nu}=\nu_{x} \partial_{x x}^{2}+\nu_{y} \partial_{y y}^{2}+\nu_{z} \partial_{z z}^{2}$, where $\nu=\left(\nu_{x}, \nu_{y}, \nu_{z}\right)$ is the 
(adimensional kinematic) viscosity. For simplicity, isotropic diffusion with $\nu_{x}=\nu_{y}=\nu_{z}=$ 1 will be considered.

The main difference between the hydrostatic Stokes equations (1)-(3) and the "classical" Stokes equations, is the disappearance of $v$ in the vertical momentum equation, which is reduced to the hydrostatic equation $\partial_{z} p=0$. In fact, owing to this reason, $v$ losses its role as a coercive variable and the formulation of (1)-(3) in a saddle-point framework is not straightforward (see Section 4). Also that reason makes more difficult the numerical approximation of (1)-(3), because an additional "hydrostatic" inf-sup condition (see Section 5) will be introduced, implying in particular instability of usual finite element spaces (like Taylor-Hood $\mathcal{P}_{2}-\mathcal{P}_{1}$ ), see section 6 below.

In short, the purpose of this paper is to delve into the analysis of the hydrostatic Stokes (or primitive) equations of the ocean and to apply the knowledge acquired to obtain some conclusions about their approximation by finite element techniques. Specifically, the stability of some (customary or new) finite element spaces, based in usual linear or quadratic polynomial approximations in conventional (structured or not) meshes, is confirmed (and denied in some cases, notably for some standard Stokes stable finite element combinations). Hence the interest of this work: opening up the possibility of approximating primitive equations with standard $\mathcal{P}_{1}, \mathcal{P}_{1}$-bubble or $\mathcal{P}_{2}$ polynomial approximations in usual not structured meshes and by using standard software tools.

This paper is organized as follows. In section 2, we give an introduction to the hydrostatic Stokes (or primitive) equations of the ocean, we fix notation and summarize previous approaches in literature. In Section 3, a first proof of well-posedness of the hydrostatic Stokes problem (1)-(3) is provided (similar to the one presented by P. Azérad in [Azé94, Azé96])). The key is the introduction of two inf-sup conditions depending on the two constraints (2) and (3), respectively. One of them plays the role of the Stokes inf-sup condition (bounding the pressure as a function of the velocity), while the other one bounds the vertical velocity as a function of the pressure. This latter "hydrostatic" inf-sup condition has no parallelism in the Stokes case and, in this sense, the hydrostatic Stokes problem needs more constraints to get stability than the Stokes one.

In Section 4, we present a second proof of the well-posedness of (1)-(3), based on the saddle-point theory for mixed finite elements (see for instance [BF91]). This proof is sharper in the sense that it illustrates the role of the hydrostatic inf-sup condition to enforce the coercivity of the bilinear form underlying to the saddle-point formulation of the hydrostatic Stokes problem.

In Section 5, the previous ideas are applied to the analysis of finite element approximations of the hydrostatic Stokes problem (1)-(3) and, in Section 6, different combinations of velocity/pressure finite elements are studied, both from the numerical analysis point of view and through numerical simulations. In fact, we prove that when $\mathbf{u}, v, p$ are approximated respectively by continuous $\mathcal{P}_{2}, \mathcal{P}_{2}$ and $\mathcal{P}_{1}$ finite elements (which will be denoted by $\left(\mathcal{P}_{2}, \mathcal{P}_{2}\right)-\mathcal{P}_{1}$ ), the resulting scheme is not stable (although it is stable for the Stokes problem). The same applies to $\left(\mathcal{P}_{1, b}, \mathcal{P}_{1, b}\right)-\mathcal{P}_{1}$. On the other hand, we show that the approximation of $\mathbf{u}, v, p$ by $\left(\mathcal{P}_{2}, \mathcal{P}_{1}\right)-\mathcal{P}_{0}$ (which is Stokes-stable, see [Ste90]) is also hydrostatic-stable and we approach numerically its accuracy order in space. Moreover, we conjecture the hydrostatic stability of $\left(\mathcal{P}_{1, b}, \mathcal{P}_{1}\right)-\mathcal{P}_{1}$ and $\left(\mathcal{P}_{2}, \mathcal{P}_{1}\right)-\mathcal{P}_{1}$ combinations in most unstructured meshes (see section 6.4), based on the Stokes-stable results provided by the authors in [GR12]. We also check numerically its accuracy order. 
Finally, in Section 7 we exploit the benefits of using the previous stable finite elements for the hydrostatic Stokes equations (1)-(3) in unstructured meshes, developing computational tests in different domains (with or without sidewall talus) and we apply mesh adaptivity techniques. Note that these adaptive techniques would not have been easy to implement for most schemes given in literature (where a vertical structured mesh is necessary).

For the purposes of scientific openness, the source code of numerical tests presented below and the graphics contained in current paper have been published, under a free license, in https://bitbucket.org/rrgalvan/hydstokes-unstructured.

\section{Preliminaries and problem setting}

Let us start delving into the primitive equations of the ocean, fixing notation and summarizing previous approaches in literature.

First of all, we fix a Cartesian system of coordinates, supposing that the domain under consideration has dimensions much shorter than the Earth's radius (a length scale not exceeding $1000 \mathrm{~km}$ is acceptable [CB09]). Moreover, taking into account the disparity between horizontal and vertical scales of large scale geophysical domains, the resulting domain, denoted by $\Omega_{\varepsilon} \subset \mathbb{R}^{3}$, is supposed to satisfy that the so-called aspect ratio

$$
\varepsilon=\frac{\text { vertical scale }}{\text { horizontal scale }} \quad \text { is very small, }
$$

for example a few $\mathrm{kms}$ over some thousand $\mathrm{kms}$, that is $\varepsilon \simeq 10^{-3}, 10^{-4}$. We denote the spatial points as $\left(\mathbf{x}, z^{\varepsilon}\right) \in \Omega_{\varepsilon}$, with $\mathbf{x}=(x, y)$.

The description of the fluid system is not complete until a relation between density and pressure is also provided. Although in the oceanic water the density is a complicated function of pressure, temperature and salinity, in this work we focus in the case of an incompressible fluid, such as pure water at ordinary pressures and temperatures, where constant density can be assumed, leaving the density-dependent case for a future work.

In this case, the set of laws corresponding to the conservation of mass and momentum are completed and can be studied separately. They constitute the Navier-Stokes equations for large scale ocean, where due to the difference between horizontal and vertical scales, anisotropic eddy viscosities (see [CB09, Ped87]) are considered, denoting them by $\nu=$ $\left(\nu_{x}, \nu_{y}, \varepsilon^{2} \nu_{z}\right)$, where $\nu_{x}, \nu_{y}$ and $\nu_{z}$ have $O(1)$ scale. The unknowns are the $3 \mathrm{D}$ velocity field, $\left(\mathbf{u}, v^{\varepsilon}\right): \Omega_{\varepsilon} \times(0, T) \longrightarrow \mathbb{R}^{3}$ and the pressure, $p: \Omega_{\varepsilon} \times(0, T) \longrightarrow \mathbb{R}$.

It is then natural to perform the geometrical scaling $z=z^{\varepsilon} / \varepsilon$ (see [AG01, BL92]), obtaining the adimensional domain (figure 1):

$$
\Omega=\left\{(\mathbf{x}, z) \in \mathbb{R}^{3} / \mathbf{x}=(x, y) \in S,-D(\mathbf{x})<z<0\right\},
$$

where $S \subset \mathbb{R}^{2}$ is a bounded domain (the surface) and $D: \bar{S} \rightarrow \mathbb{R}_{+}$is a function describing the bottom depth. Here, the rigid lid hypothesis has been assumed: the true free surface of the ocean has been approximated by means of a flat averaged surface. We will denote $\Gamma_{S}=\bar{S} \times\{0\}$ the surface boundary, $\Gamma_{b}=\{(\mathbf{x},-D(\mathbf{x})) / \mathbf{x}=(x, y) \in S\}$ the bottom boundary and $\Gamma_{l}=\{(\mathbf{x}, z) / \mathbf{x} \in \partial S,-D(\mathbf{x})<z<0\}$ the lateral walls (if it exists). 


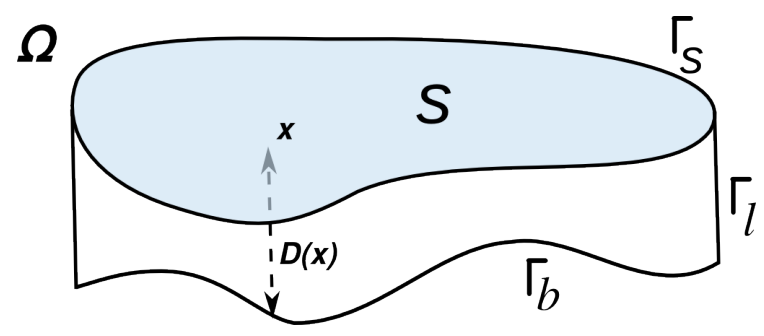

Figure 1: Adimensional rigid-lid domain $\Omega$

Thus, if the kinematic scaling $v=v^{\varepsilon} / \varepsilon$ is performed, one has the next anisotropic Navier-Stokes equations in the time-space domain $(0, T) \times \Omega$ :

$$
\begin{aligned}
\partial_{t} \mathbf{u}+\left(\mathbf{u} \cdot \nabla_{\mathbf{x}}\right) \mathbf{u}+v \partial_{z} \mathbf{u}-\Delta_{\nu} \mathbf{u}+\nabla_{\mathbf{x}} p & =\mathbf{f}, \\
\varepsilon^{2}\left\{\partial_{t} v+\left(\mathbf{u} \cdot \nabla_{\mathbf{x}}\right) v+v \partial_{z} v-\Delta_{\nu} v\right\}+\partial_{z} p & =g, \\
\nabla_{\mathbf{x}} \cdot \mathbf{u}+\partial_{z} v & =0 .
\end{aligned}
$$

The term $\mathbf{f}=\left(f_{1}, f_{2}\right)^{t}$ models other phenomena, like the Coriolis acceleration (in this case $\mathbf{f}=\mathbf{f}(\mathbf{u}))$. The term $g$ involves both the force due to the gravity and other effects like explicit Coriolis (of order $\varepsilon$, cf. [AG01]).

In this paper, Coriolis effect will not be considered (because this term is linear and orthogonal to the flow, hence results presented below are not affected). Then $g$, represents only the force due to gravity that, as is usual, will be written in the potential form $g=\partial_{z} \varphi$, for a given function $\varphi$, which can be incorporated to the pressure term (redefining $p=p-\varphi)$. Consequently, we can take $g=0$ in (6).

The system is endowed with initial conditions $\left.(\mathbf{u}, v)\right|_{t=0}=\left(\mathbf{u}_{0}, v_{0}\right)$ and adequate boundary conditions. On the surface $\Gamma_{s}$, we will consider the rigid lid hypothesis and a winddriven condition (by the stress of a function $\mathbf{g}_{s}$ ), while adherence conditions are considered for $\mathbf{u}$ and $v$ on the bottom $\Gamma_{b}$ and for $\mathbf{u}$ on the sidewall $\Gamma_{l}$ (if it exists):

$$
\begin{array}{r}
\left.\nu_{z} \partial_{z} \mathbf{u}\right|_{\Gamma_{S}}=\mathbf{g}_{s},\left.\quad v\right|_{\Gamma_{S}}=0 \\
\left.\mathbf{u}\right|_{\Gamma_{b} \cup \Gamma_{l}}=0,\left.\quad v\right|_{\Gamma_{b}}=0, \\
\left.\nabla v \cdot \mathbf{n}_{\mathbf{x}}\right|_{\Gamma_{l}}=0 .
\end{array}
$$

Here $\mathbf{n}_{\mathbf{x}}$ is the horizontal component of the outwards normal vector. The choice of the slip condition (10) on $\Gamma_{l}$, instead of a Dirichlet one, prevents boundary layers on $\Gamma_{l}$, which may appear for small $\varepsilon$ due to the loss of regularity of the vertical velocity which follows from the $\varepsilon^{2}$-terms in (6).

In this work, instead of supposing $\varepsilon$ small, we will center directly in the limit case $\varepsilon=0$, arriving at the so-called hydrostatic Navier-Stokes equations or primitive equations (of the Ocean) in $(0, T) \times \Omega$ :

$$
\begin{aligned}
\partial_{t} \mathbf{u}+\left(\mathbf{u} \cdot \nabla_{\mathbf{x}}\right) \mathbf{u}+v \partial_{z} \mathbf{u}-\Delta_{\nu} \mathbf{u}+\nabla_{\mathbf{x}} p & =\mathbf{f}, \\
\partial_{z} p & =0, \\
\nabla_{\mathbf{x}} \cdot \mathbf{u}+\partial_{z} v & =0,
\end{aligned}
$$


endowed with boundary conditions (8) and (9). Note that the vertical velocity $v$ loses its regularity in the $\mathbf{x}$-direction and then boundary conditions on sidewalls (10) disappear. Equations (11)-(13) can be obtained as limit of the anisotropic Navier-Stokes equations (5) $-(7)$ when $\varepsilon$ tends to zero. This fact is justified on rigorous mathematical grounds in [BL92, AG01].

Many works about existence and regularity results of (11)-(13) are based on replacing this problem by the next (equivalent) reduced problem: find $\mathbf{u}: \Omega \times(0, T) \rightarrow \mathbb{R}^{2}$, the horizontal velocity and $p_{s}: S \times(0, T) \rightarrow \mathbb{R}$ an (artificial) surface pressure, satisfying

$$
\begin{aligned}
\partial_{t} \mathbf{u}+\left(\mathbf{u} \cdot \nabla_{\mathbf{x}}\right) \mathbf{u}+v \partial_{z} \mathbf{u}-\Delta_{\nu} \mathbf{u}+\nabla_{\mathbf{x}} p_{s} & =\mathbf{f}, & & \text { in }(0, T) \times \Omega, \\
\nabla_{\mathbf{x}} \cdot\langle\mathbf{u}\rangle & =0, & & \text { in }(0, T) \times S, \\
\left.\mathbf{u}\right|_{\Gamma_{b} \cup \Gamma_{l}}=0,\left.\quad \nu_{z} \partial_{z} \mathbf{u}\right|_{\Gamma_{S}} & =\mathbf{g}_{s}, & &
\end{aligned}
$$

where $v$ and $\langle\mathbf{u}\rangle$ are defined by the vertical integrations:

$$
v(\mathbf{x}, z, t)=\int_{z}^{0} \nabla_{\mathbf{x}} \cdot \mathbf{u}\left(\mathbf{x}, z^{\prime}, t\right) d z^{\prime}, \quad\langle\mathbf{u}\rangle(\mathbf{x}, t)=\int_{-D(\mathbf{x})}^{0} \mathbf{u}(\mathbf{x}, z, t) d z .
$$

The existence of weak solutions of (14)-(16) was obtained in [LTW92b]. Further extensions can be found, for instance, in [CG00, Ort04] and references therein. With respect to regularity results, the existence of strong solutions (with $H^{2}(\Omega)$-regularity for the horizontal velocity) was treated in [Zia95] for the linear stationary case. For the nonlinear time-dependent problem, the existence and uniqueness of local in time strong solutions is given in [GMR01]. Finally, in [CT07] it is shown that this strong solution is global in time, imposing interesting physical boundary conditions which include wind driven stress on the surface and slip friction on the bottom.

In practice reduced problem (14)-(16) presents some advantages over the original hydrostatic one (11)-(13); the vertical velocity can be computed as a function of $\nabla_{\mathbf{x}} \cdot \mathbf{u}$, hence the number of unknowns is reduced, and the dimension of the pressure is also reduced. Therefore, equations (14)-(16) has been the preferred framework for developing numerical schemes, see e.g. [CG00, CR, CR05, GR05, CGS12].

But, in order to approximate by finite elements, the reduced formulation (14)-(16) is non-local (requiring the calculus of global $z$-integrals) which imposes a strong vertical structure to the mesh and, in practice, makes difficult to use "standard" finite element software tools. From this point of view, the original (local) hydrostatic Navier-Stokes problem (11)-(13) has the advantage of allowing one to approximate by standard unstructured finite element meshes and to use a mesh adaptive tool. The drawback is that (as occurs for the anisotropic Navier-Stokes system (5)-(7) when $\varepsilon \rightarrow 0$ ) the strong anisotropy of the hydrostatic equations (11)-(13) imply that many finite element pairs which are stable for the Navier-Stokes problem, for instance $\mathcal{P}_{2}-\mathcal{P}_{1}$ or $\mathcal{P}_{1, b}-\mathcal{P}_{1}$, are not stable in the hydrostatic case (see Section 6).

This paper focus on the theoretic and numerical study of the linear steady case (1)-(3), which arises in linearized time-discrete schemes of (11)-(13). For example, considering a semi-implicit time scheme, implicit for the diffusion and explicit for the convection, we arrive at equations like

$$
\alpha \mathbf{u}-\Delta \mathbf{u}+\nabla_{\mathbf{x}} p=\mathbf{F} \quad \text { in } \Omega
$$


where $\alpha=1 / d t$, with $d t>0$ the time step, and the explicit approximations are collected in $\mathbf{F}$. If $\alpha=0$ this equation coincides with (1). The case $\alpha \neq 0$ does not introduce any additional difficulty with respect to the results of this paper.

\section{Well-posedness of the hydrostatic Stokes problem: a first proof}

In this section, well-posedness of problem (1)-(3), (8)-(9) is shown, by means of an argument introduced by P. Azerad in [Azé94, Azé96]. Let us define spaces

$$
\begin{aligned}
\mathbf{H}_{b, l}^{1}(\Omega) & =\left\{\mathbf{u} \in H^{1}(\Omega)^{2} /\left.\mathbf{u}\right|_{\Gamma_{b} \cup \Gamma_{l}}=0\right\} \\
H_{z, 0}^{1}(\Omega) & =\left\{v \in L^{2}(\Omega) / \partial_{z} v \in L^{2}(\Omega),\left.v\right|_{\Gamma_{S} \cup \Gamma_{b}}=0\right\}, \\
L_{0}^{2}(\Omega) & =\left\{p \in L^{2}(\Omega) / \int_{\Omega} p=0\right\}
\end{aligned}
$$

and denote

$$
\mathbf{U}=\mathbf{H}_{b, l}^{1}(\Omega), \quad V=H_{z, 0}^{1}(\Omega), \quad P=L_{0}^{2}(\Omega) .
$$

$\mathbf{H}_{b, l}^{1}(\Omega)$ is endowed with the $L^{2}(\Omega)$-norm of the gradient

$$
\|\nabla \mathbf{u}\|=\sqrt{\left\|\partial_{x} u_{1}\right\|^{2}+\left\|\partial_{y} u_{1}\right\|^{2}+\left\|\partial_{x} u_{2}\right\|^{2}+\left\|\partial_{y} u_{2}\right\|^{2}}
$$

(hereafter $\|\cdot\|$ denotes the $L^{2}(\Omega)$-norm) which is equivalent to the standard $H^{1}(\Omega)$-norm, owing to the homogeneous Dirichlet conditions for $\mathbf{u}$ on $\Gamma_{l} \cup \Gamma_{b}$. Furthermore $H_{z, 0}^{1}(\Omega)$ is endowed with $\left\|\partial_{z} v\right\|$, which is a norm owing to the homogeneous Dirichlet condition of $v$ on $\Gamma_{s} \cup \Gamma_{b}$ and the vertical Poincaré inequality:

$$
\|v\| \leq C\left\|\partial_{z} v\right\| \quad \forall v \in H_{z, 0}^{1}(\Omega)
$$

Definition 1. It will be said that $(\mathbf{u}, v, p) \in \mathbf{U} \times V \times P$ is a weak solution of the hydrostatic Stokes problem (1)-(3), (8)-(9) if

$$
\begin{aligned}
(\nabla \mathbf{u}, \nabla \overline{\mathbf{u}})-\left(p, \nabla_{\mathbf{x}} \overline{\mathbf{u}}\right) & =\langle\mathbf{f}, \overline{\mathbf{u}}\rangle, \quad \forall \mathbf{u} \in \mathbf{U}, \\
\left(p, \partial_{z} \bar{v}\right) & =0, \quad \forall v \in V, \\
(\nabla \cdot(\mathbf{u}, v), \bar{p}) & =0, \quad \forall p \in P .
\end{aligned}
$$

Hereafter, $(\cdot, \cdot)$ denotes the $L^{2}(\Omega)$ inner product and $\langle\cdot, \cdot\rangle$ the $\mathbf{H}_{b, l}^{-1}(\Omega) \times \mathbf{H}_{b, l}^{1}(\Omega)$ duality. Note that, in the variational formulation (18)-(20), $\mathbf{u} \in \mathbf{U}$ is the only "coercive" variable, hence $v \in V$ and $p \in P$ play the role of two Lagrange multipliers related to the hydrostatic and incompressibility constraints (2) and (3), respectively. It is then natural to consider the following (inf-sup) conditions:

$$
\begin{aligned}
\sup _{0 \neq(\mathbf{u}, v) \in \mathbf{U} \times V} \frac{(\nabla \cdot(\mathbf{u}, v), p)}{\left\|\nabla \mathbf{u}, \partial_{z} v\right\|} \geq \beta^{P}\|p\| & \forall p \in P, \\
\sup _{0 \neq p \in P} \frac{\left(\partial_{z} v, p\right)}{\|p\|} \geq \beta^{V}\left\|\partial_{z} v\right\| & \forall v \in V,
\end{aligned}
$$

where $\beta^{P}, \beta^{V}>0$ and $\left\|\nabla \mathbf{u}, \partial_{z} v\right\|=\sqrt{\|\nabla \mathbf{u}\|^{2}+\left\|\partial_{z} v\right\|^{2}}$ is the euclidean norm related to the vectorial space $\mathbf{H}_{b, l}^{1}(\Omega) \times H_{z, 0}^{1}(\Omega)$. 
Remark 1. Condition $(I S)^{P}$ is equivalent to (see for instance [BF91]): for each $p \in P$, there exists $(\widetilde{\mathbf{u}}, \widetilde{v}) \in \mathbf{U} \times V$ such that

$$
(\nabla \cdot(\widetilde{\mathbf{u}}, \widetilde{v}), p) \geq \beta^{P}\|p\|^{2} \quad \text { and } \quad\left\|\nabla \widetilde{\mathbf{u}}, \partial_{z} \widetilde{v}\right\| \leq\|p\| .
$$

Condition $(I S)^{V}$ is equivalent to: for each $v \in V$, there exists $\widetilde{p} \in P$ such that

$$
\left(\partial_{z} v, \widetilde{p}\right) \geq \beta^{V}\left\|\partial_{z} v\right\|^{2} \quad \text { and } \quad\|\widetilde{p}\| \leq\left\|\partial_{z} v\right\| .
$$

Remark 2. Inf-sup condition $(I S)^{P}$ is rather similar to the well-known inf-sup Stokes condition: there exits $\beta>0$ such that

$$
\sup _{0 \neq(\mathbf{u}, v) \in H_{0}^{1}(\Omega)^{3}} \frac{(\nabla \cdot(\mathbf{u}, v), p)}{\|\nabla \mathbf{u}, \nabla v\|} \geq \beta\|p\| \quad \forall p \in P .
$$

The difference is that in $(I S)^{P}$ the $H^{1}$-norm of $v$ is replaced by the $H_{z}^{1}$-norm $\left\|\partial_{z} v\right\|$.

Lemma 1. If $\mathbf{U}, V$ and $P$ are defined by (17), then there exist two positive constants, $\beta^{P}$ and $\beta^{V}>0$, such that $(I S)^{P}$ and $(I S)^{V}$ hold. In fact, $\beta^{P}$ can be taken as the Stokes inf-sup constant and $\beta^{V}=1$.

Proof. $(I S)^{P}$ is consequence of the Stokes inf-sup $(I S)$. Indeed, given $p \in P=L_{0}^{2}(\Omega)$,

$$
\sup _{0 \neq(\mathbf{u}, v) \in \mathbf{U} \times V} \frac{(\nabla \cdot(\mathbf{u}, v), p)}{\left\|\nabla \mathbf{u}, \partial_{z} v\right\|} \geq \sup _{0 \neq(\mathbf{u}, v) \in H_{0}^{1}(\Omega)^{3}} \frac{(\nabla \cdot(\mathbf{u}, v), p)}{\|\nabla \mathbf{u}, \nabla v\|} \geq \beta\|p\|,
$$

hence $(I S)^{P}$ holds with $\beta^{P}=\beta$ (the Stokes inf-sup constant).

On the other hand, $(I S)^{V}$ can be obtained directly using that $\partial_{z} v \in P$ for each $v \in V$. Indeed, taking $\widetilde{p}=\partial_{z} v$, then $\widetilde{p} \in L^{2}(\Omega)$ and

$$
\int_{\Omega} \tilde{p}=\int_{S} \int_{-D(\mathbf{x})}^{0} \partial_{z} v(\mathbf{x}, z) d z d \mathbf{x}=\int_{S}(v(\mathbf{x}, 0)-v(\mathbf{x},-D(\mathbf{x}))) d \mathbf{x}=0,
$$

where the last equality is a consequence of the Dirichlet conditions for $v$ given in (8) and (9). Then, we have found $\widetilde{p} \in P$ such that $\left(\widetilde{p}, \partial_{z} v\right)=\left\|\partial_{z} v\right\|^{2}$ and $\|\widetilde{p}\|=\left\|\partial_{z} v\right\|$, what implies $(I S)^{V}$ (with $\beta^{V}=1$ ).

Lemma 2. The following inequality holds

$$
\left\|\nabla_{\mathbf{x}} \cdot \mathbf{u}\right\| \leq\left\|\nabla_{\mathbf{x}} \mathbf{u}\right\|, \quad \forall \mathbf{u} \in \boldsymbol{H}_{b, l}^{1}(\Omega) .
$$

The proof of this inequality is based on the integration by parts formula (recall that $\left.\mathbf{x}=(x, y)^{t}\right)$

$$
\int_{\Omega} \partial_{x} u_{1} \partial_{y} u_{2}=\int_{\Omega} \partial_{y} u_{1} \partial_{x} u_{2}
$$

which is valid for regular functions vanishing on $\Gamma_{b} \cup \Gamma_{l}$.

Now, we are in position to prove existence, uniqueness and energy estimates for the hydrostatic Stokes problem (1)-(3), (8)-(9).

Theorem 3. The following statements are equivalent: 
1. U, $V$ and $P$ satisfy $(I S)^{P}$ and $(I S)^{V}$.

2. Problem (1)-(3), (8)-(9) is well-posed in $\mathbf{U} \times V \times P$.

In this case, there exists an unique weak solution $(\mathbf{u}, v, p) \in \mathbf{U} \times V \times P$ of (1)-(3), (8)-(9) and the following estimates hold:

$$
\|\nabla \mathbf{u}\| \leq\|\mathbf{f}\|_{\mathbf{U}^{\prime}}, \quad\left\|\partial_{z} v\right\| \leq \frac{1}{\beta^{V}}\|\mathbf{f}\|_{\mathbf{U}^{\prime}}, \quad\|p\| \leq \frac{2}{\beta^{P}}\|\mathbf{f}\|_{\mathbf{U}^{\prime}}
$$

where $\|\mathbf{f}\|_{\mathbf{U}^{\prime}}$ denotes the dual norm of $\mathbf{U}=\boldsymbol{H}_{b, l}^{1}(\Omega)$.

Proof. We split the proof into four steps.

Step 1. Rewriting the variational formulation (18)-(20).

We consider the Hilbert space $\mathbf{X}=\mathbf{H}_{b, l}^{1}(\Omega) \times H_{z, 0}^{1}(\Omega) \times L_{0}^{2}(\Omega)$ endowed with the norm $\|\boldsymbol{\chi}\|_{\mathbf{X}}^{2}=\|\nabla \mathbf{u}\|^{2}+\left\|\partial_{z} v\right\|^{2}+\|p\|^{2}$ for each $\boldsymbol{\chi}=(\mathbf{u}, v, p) \in \mathbf{X}$. Let us define the following bilinear and linear continuous forms, for each $\boldsymbol{\chi}=(\mathbf{u}, v, p) \in \mathbf{X}$ and $\bar{\chi}=(\overline{\mathbf{u}}, \bar{v}, \bar{p}) \in \mathbf{X}$ :

$$
\begin{aligned}
\mathcal{A}(\boldsymbol{\chi}, \overline{\boldsymbol{\chi}}) & =(\nabla \mathbf{u}, \nabla \overline{\mathbf{u}})-(p, \nabla \cdot(\overline{\mathbf{u}}, \bar{v}))-(\nabla \cdot(\mathbf{u}, v), \bar{p}), \\
\mathcal{L}(\overline{\boldsymbol{\chi}}) & =\langle\mathbf{f}, \overline{\mathbf{u}}\rangle_{\mathbf{U}^{\prime}, \mathbf{U}}
\end{aligned}
$$

Then, weak formulation (18)-(20) can be written as:

$$
\text { find } \chi \in \mathbf{X} \quad \text { such that } \mathcal{A}(\chi, \bar{\chi})=\mathcal{L}(\bar{\chi}) \quad \forall \bar{\chi} \in \mathbf{X} \text {. }
$$

Since $\mathcal{A}(\cdot, \cdot)$ is symmetric, for the existence and uniqueness of (18)-(20) (i.e. of (24)) it is sufficient (and necessary) to show the next generalized elliptic hypothesis of the socalled Banach-Necas-Babuska's Theorem (see for instance [EG04, Theorem 2.6]): there exists $\gamma>0$ such that

$$
\sup _{0 \neq \overline{\boldsymbol{\chi}} \in \mathbf{X}} \frac{\mathcal{A}(\boldsymbol{\chi}, \overline{\boldsymbol{\chi}})}{\|\overline{\boldsymbol{\chi}}\|_{\mathbf{X}}} \geq \gamma\|\chi\|_{\mathbf{X}} \quad \forall \chi \in \mathbf{X}
$$

Step 2. Proof of the abstract inf-sup condition (25).

Let $\boldsymbol{\chi}=(\mathbf{u}, v, p) \in \mathbf{X}$. If we consider $\overline{\boldsymbol{\chi}}_{1}=(\mathbf{u}, v,-p)$, then

$$
\mathcal{A}\left(\boldsymbol{\chi}, \overline{\boldsymbol{\chi}}_{1}\right)=(\nabla \mathbf{u}, \nabla \mathbf{u})-(\nabla \cdot(\mathbf{u}, v), p)+(\nabla \cdot(\mathbf{u}, v), p)=\|\nabla \mathbf{u}\|^{2} .
$$

Owing to $(I S)^{P}$, fixed $p \in P$, there exists $(\widetilde{\mathbf{u}}, \widetilde{v}) \in \mathbf{U} \times V$ such that

$$
\left\|\nabla \widetilde{\mathbf{u}}, \partial_{z} \widetilde{v}\right\| \leq\|p\| \quad \text { and } \quad(\nabla \cdot(\widetilde{\mathbf{u}}, \widetilde{v}), p) \geq \beta^{P}\|p\|^{2} .
$$

Then, if we define $\bar{\chi}_{2}=(-\widetilde{\mathbf{u}},-\widetilde{v}, 0) \in \mathbf{X}$,

$$
\begin{aligned}
\mathcal{A}\left(\boldsymbol{\chi}, \overline{\boldsymbol{\chi}}_{2}\right)=-(\nabla \mathbf{u}, \nabla \widetilde{\mathbf{u}})+(\nabla \cdot(\widetilde{\mathbf{u}}, \widetilde{v}), p) \geq-\|\nabla \mathbf{u}\|\|\nabla \widetilde{\mathbf{u}}\|+\beta^{P}\|p\|^{2} & \\
& \geq-\|\nabla \mathbf{u}\|\|p\|+\beta^{P}\|p\|^{2} \geq \frac{\beta^{P}}{2}\|p\|^{2}-\frac{1}{2 \beta^{P}}\|\nabla \mathbf{u}\|^{2} .
\end{aligned}
$$

Now, owing to $(I S)^{V}$, fixed $v \in V$, there exists $\widetilde{p} \in P$ such that

$$
\|\widetilde{p}\| \leq\left\|\partial_{z} v\right\| \quad \text { and } \quad\left(\partial_{z} v, \widetilde{p}\right) \geq \beta^{V}\left\|\partial_{z} v\right\|^{2} .
$$


Then, defining $\bar{\chi}_{3}=(0,0,-\widetilde{p}) \in \mathbf{X}$,

$$
\begin{aligned}
\mathcal{A}\left(\boldsymbol{\chi}, \overline{\boldsymbol{\chi}}_{3}\right) & =\left(\nabla_{\mathbf{x}} \cdot \mathbf{u}, \widetilde{p}\right)+\left(\partial_{z} v, \widetilde{p}\right) \geq-\left\|\nabla_{\mathbf{x}} \cdot \mathbf{u}\right\|\|\widetilde{p}\|+\beta^{V}\left\|\partial_{z} v\right\|^{2} \\
& \geq-\left\|\nabla_{\mathbf{x}} \cdot \mathbf{u}\right\|\left\|\partial_{z} v\right\|+\beta^{V}\left\|\partial_{z} v\right\|^{2} \geq \frac{\beta^{V}}{2}\left\|\partial_{z} v\right\|^{2}-\frac{1}{2 \beta^{V}}\|\nabla \mathbf{u}\|^{2},
\end{aligned}
$$

where in the last inequality Lemma 2 has been applied.

Taking $\bar{\chi}=\alpha \bar{\chi}_{1}+\bar{\chi}_{2}+\bar{\chi}_{3}$, with $\alpha>\frac{1}{2 \beta^{P}}+\frac{1}{2 \beta^{V}}$ and considering (26)-(28), we get

$$
\begin{aligned}
\mathcal{A}(\boldsymbol{\chi}, \overline{\boldsymbol{\chi}}) \geq\left(\alpha-\frac{1}{2 \beta^{P}}-\frac{1}{2 \beta^{V}}\right)\|\nabla \mathbf{u}\|^{2}+\frac{\beta^{V}}{2}\left\|\partial_{z} v\right\|^{2}+\frac{\beta^{P}}{2}\|p\|^{2} & \\
& \geq \lambda\left(\|\nabla \mathbf{u}\|^{2}+\left\|\partial_{z} v\right\|^{2}+\|p\|^{2}\right)=\lambda\|\chi\|_{\mathbf{X}}^{2}
\end{aligned}
$$

where

$$
\lambda=\min \left\{\alpha-\frac{1}{2 \beta^{P}}-\frac{1}{2 \beta^{V}}, \frac{\beta^{P}}{2}, \frac{\beta^{V}}{2}\right\} .
$$

On the other hand:

$$
\begin{gathered}
\|\overline{\boldsymbol{\chi}}\|_{\mathbf{X}}^{2}=\|(\alpha \mathbf{u}-\widetilde{\mathbf{u}}, \alpha v-\widetilde{v},-\alpha p-\widetilde{p})\|_{\mathbf{X}}^{2}=\|\nabla(\alpha \mathbf{u}-\widetilde{\mathbf{u}})\|^{2}+\left\|\partial_{z}(\alpha v-\widetilde{v})\right\|^{2}+\|\alpha p+\widetilde{p}\|^{2} \\
\leq 2\left(\alpha^{2}\left\|\nabla \mathbf{u}, \partial_{z} v, p\right\|^{2}+\left\|\nabla \widetilde{\mathbf{u}}, \partial_{z} \widetilde{v}, \widetilde{p}\right\|^{2}\right) \leq 2\left(\alpha^{2}+1\right)\left\|\nabla \mathbf{u}, \partial_{z} v, p\right\|^{2} \leq 2\left(\alpha^{2}+1\right)\|\chi\|_{\mathbf{X}}^{2}
\end{gathered}
$$

Then (25) holds for $\gamma=\lambda / \sqrt{2\left(\alpha^{2}+1\right)}$.

Step 3. Proof of stability estimates (21).

The Banach-Necas-Babuska's theorem, which has been used for the existence of solution of (25), also provides the "vectorial" estimate $\|\bar{\chi}\|_{\mathbf{X}} \leq\|\mathcal{L}\| / \gamma$ (see for instance [EG04, Theorem 2.6]). But, we will go beyond, and show a priori estimates (21), where the dependence on $\beta^{P}$ and $\beta^{V}$ is explicit.

Let $\boldsymbol{\chi}=(\mathbf{u}, v, p)$ be the solution of (18)-(20), i.e. of (24). Taking $\overline{\boldsymbol{\chi}}=(\mathbf{u}, v,-p)$ in (24), one has $\|\nabla \mathbf{u}\|^{2}=\langle\mathbf{f}, \mathbf{u}\rangle_{\mathbf{U}^{\prime}, \mathbf{U}} \leq\|\mathbf{f}\|_{\mathbf{U}^{\prime}}\|\nabla \mathbf{u}\|$, and then

$$
\|\nabla \mathbf{u}\| \leq\|\mathbf{f}\|_{\mathbf{U}^{\prime}}
$$

Using the latter inequality, $(I S)^{V}$ and taking $\bar{\chi}=(0,0, \bar{p})$ in $(24)$ :

$$
\begin{aligned}
\left\|\partial_{z} v\right\| \leq \frac{1}{\beta^{V}} \sup _{0 \neq \bar{p} \in P} \frac{\left(\partial_{z} v, \bar{p}\right)}{\|\bar{p}\|} & =\frac{1}{\beta^{V}} \sup _{0 \neq \bar{p} \in P} \frac{\left(-\nabla_{\mathbf{x}} \cdot \mathbf{u}, \bar{p}\right)}{\|\bar{p}\|} \\
& =\frac{1}{\beta^{V}}\left\|\nabla_{\mathbf{x}} \cdot \mathbf{u}\right\| \leq \frac{1}{\beta^{V}}\|\mathbf{f}\|_{\mathbf{U}^{\prime}}
\end{aligned}
$$

where in last equality we use that the supremun is taken in $\bar{p}=-\nabla_{\mathbf{x}} \cdot \mathbf{u}$.

Finally, using $(I S)^{P}$ and taking $\bar{\chi}=(\overline{\mathbf{u}}, \bar{v}, 0)$ in $(24)$,

$$
\begin{aligned}
\|p\| \leq \frac{1}{\beta^{P}} \sup _{0 \neq(\overline{\mathbf{u}}, \bar{v}) \in \mathbf{U} \times V} \frac{(\nabla \cdot(\overline{\mathbf{u}}, \bar{v}), p)}{\left\|\nabla \overline{\mathbf{u}}, \partial_{z} \bar{v}\right\|} & \\
=\frac{1}{\beta^{P}} \sup _{0 \neq(\overline{\mathbf{u}}, \bar{v}) \in \mathbf{U} \times V} \frac{(\nabla \mathbf{u}, \nabla \overline{\mathbf{u}})-\langle\mathbf{f}, \overline{\mathbf{u}}\rangle_{\mathbf{H}_{b, l}^{-1}(\Omega), \mathbf{H}_{b, l}^{1}(\Omega)}}{\left\|\nabla \overline{\mathbf{u}}, \partial_{z} \bar{v}\right\|} & \leq \frac{1}{\beta^{P}}\left(\|\nabla \mathbf{u}\|+\|\mathbf{f}\|_{\mathbf{U}^{\prime}}\right) \leq \frac{2}{\beta^{P}}\|\mathbf{f}\|_{\mathbf{U}^{\prime}}
\end{aligned}
$$


where again (29) has been used in the last inequality.

Step 4. The reciprocal argument.

Reciprocally we will show that inf-sup condition $(25)$ implies $(I S)^{P}$ and $(I S)^{V}$. In fact given $p \in P$, let $\chi=(0,0, p) \in \mathbf{X}$, then $\mathcal{A}(\chi, \bar{\chi})=-(\nabla \cdot(\overline{\mathbf{u}}, \bar{v}), p)$ for all $\bar{\chi}=(\overline{\mathbf{u}}, \bar{v}, \bar{p}) \in \mathbf{X}$. Taking into account (25), fixed previous $\chi=(0,0, p)$, there exists $\widetilde{\chi}=(\widetilde{\mathbf{u}}, \widetilde{v}, \widetilde{p}) \in \mathbf{X}$ :

$$
\|\widetilde{\chi}\|_{\mathbf{X}} \leq\|\chi\|_{\mathbf{X}}=\|p\| \quad \text { and } \quad \mathcal{A}(\chi, \widetilde{\chi}) \geq \gamma\|\chi\|_{\mathbf{X}}^{2}=\gamma\|p\|^{2} .
$$

In particular,

$$
\left\|\nabla \widetilde{\mathbf{u}}, \partial_{z} \widetilde{v}\right\| \leq\|p\| \quad \text { and } \quad-(\nabla \cdot(\widetilde{\mathbf{u}}, \widetilde{v}), p) \geq \gamma\|p\|^{2},
$$

hence $(I S)^{P}$ holds (with $\beta^{P}=\gamma$ ).

On the other hand, given $v \in V$, let $\chi=(0, v, 0) \in \mathbf{X}$, then $\mathcal{A}(\boldsymbol{\chi}, \overline{\boldsymbol{\chi}})=-\left(\partial_{z} v, \bar{p}\right)$ for all $\bar{\chi}=(\overline{\mathbf{u}}, \bar{v}, \bar{p}) \in \mathbf{X}$. Now (25) implies that there exists $\widetilde{\chi}=(\widetilde{\mathbf{u}}, \widetilde{v}, \widetilde{p}) \in \mathbf{X}$ such that

$$
\|\widetilde{p}\| \leq\left\|\partial_{z} v\right\| \quad \text { and } \quad-\left(\partial_{z} v, \widetilde{p}\right) \geq \gamma\left\|\partial_{z} v\right\|^{2},
$$

hence $(I S)^{V}$ holds (with $\beta^{V}=\gamma$ ).

Remark 3. Condition $(I S)^{P}$ is similar to the Stokes inf-sup condition $(I S)$ and plays a similar role in obtaining estimates for the pressure as a function of the velocity space. But condition $(I S)^{V}$ provides $H_{z}^{1}$ estimates for the vertical velocity as a function of the pressure space, which has no parallel in the Stokes problem.

\section{A new proof using the saddle point framework}

Another proof of Theorem 3, which is more descriptive about the paper of $(I S)^{V}$, can be reached via the saddle point framework (see e.g. [BF91]). Let $\mathbf{W}=\mathbf{U} \times V$ endowed with the norm $\|\mathbf{w}\|_{\mathbf{w}}=\left\|\nabla \mathbf{u}, \partial_{z} v\right\|$. Let us define, for each $\mathbf{w}=(\mathbf{u}, v)$ and $\overline{\mathbf{w}}=(\overline{\mathbf{u}}, \bar{v}) \in \mathbf{W}$ :

$$
\begin{array}{ll}
a: \mathbf{W} \times \mathbf{W} \rightarrow \mathbb{R}, & a(\mathbf{w}, \overline{\mathbf{w}})=(\nabla \mathbf{u}, \nabla \overline{\mathbf{u}}) \\
b: \mathbf{W} \times P \rightarrow \mathbb{R}, & b(\mathbf{w}, p)=-\left(\nabla_{\mathbf{x}} \cdot \mathbf{u}+\partial_{z} v, p\right) \\
B: \mathbf{W} \rightarrow P^{\prime}, B^{t}: P \rightarrow \mathbf{W}^{\prime}, & \langle B \mathbf{w}, p\rangle_{P^{\prime}, P}=b(\mathbf{w}, p)=\left\langle B^{t} p, \mathbf{w}\right\rangle_{\mathbf{W}^{\prime}, \mathbf{W}}
\end{array}
$$

Obviously, $a(\cdot, \cdot)$ and $b(\cdot, \cdot)$ are bilinear continuous forms and $a(\cdot, \cdot)$ is symmetric but not $\mathbf{W}$-elliptic. The variational problem (18)-(20) can be rewritten as: find $\mathbf{w} \in \mathbf{W}$ and $p \in P$ such that

$$
\begin{aligned}
a(\mathbf{w}, \overline{\mathbf{w}})+b(\overline{\mathbf{w}}, p) & =\left\langle(\mathbf{f}, 0)^{t}, \overline{\mathbf{w}}\right\rangle_{\mathbf{W}^{\prime}, \mathbf{W}} \quad \forall \overline{\mathbf{w}} \in \mathbf{W}, \\
b(\mathbf{w}, \bar{p}) & =0 \quad \forall \bar{p} \in P,
\end{aligned}
$$

where $\left\langle(\mathbf{f}, 0)^{t}, \overline{\mathbf{w}}\right\rangle_{\mathbf{W}^{\prime}, \mathbf{W}}=\langle\mathbf{f}, \overline{\mathbf{u}}\rangle_{\mathbf{U}^{\prime}, \mathbf{U}}$. We state a well-known result about sufficient conditions of existence and uniqueness of (30)-(31), see [BF91, Propositions 1.1 to 1.3].

Lemma 4. One has existence and uniqueness of solution of (30)-(31) in $\mathbf{W} \times\left(P / \operatorname{ker}\left(B^{t}\right)\right)$ assuming the following two conditions: 
1. $a(\cdot, \cdot)$ is coercive on $\operatorname{ker} B$, i.e., there exists $\alpha_{0}>0$ such that

$$
a\left(\mathbf{w}_{0}, \mathbf{w}_{0}\right) \geq \alpha_{0}\left\|\mathbf{w}_{0}\right\|_{\mathbf{W}}^{2}, \quad \forall \mathbf{w}_{0} \in \operatorname{ker}(B) .
$$

2. $\operatorname{Img}(B)$ is closed in $P^{\prime}$ or, equivalently:

$$
\sup _{\mathbf{w} \in \mathbf{W} \backslash\{0\}} \frac{b(\mathbf{w}, p)}{\|\mathbf{w}\|_{\mathbf{w}}} \geq \beta_{0}\|p\|_{P / \operatorname{ker}\left(B^{t}\right)}, \quad \forall p \in P .
$$

Moreover, in this case, the following estimates hold:

$$
\begin{aligned}
& \|\mathbf{w}\|_{\mathbf{W}} \leq \frac{1}{\alpha_{0}}\|\mathbf{f}\|_{\mathbf{U}^{\prime}} \\
& \|p\|_{P / \operatorname{ker}\left(B^{t}\right)} \leq \frac{1}{\beta_{0}}\left(1+\frac{\|a\|}{\alpha_{0}}\right)\|\mathbf{f}\|_{\mathbf{U}^{\prime}} .
\end{aligned}
$$

In particular, there exists an unique $\mathbf{w} \in \mathbf{W}$, while $p$ is uniquely defined excepting the addition of elements of $\operatorname{ker}\left(B^{t}\right)$.

Corollary 5. One has existence and uniqueness of solution of (30)-(31) in $\mathbf{W} \times P$.

Proof. It suffices to apply the above Lemma, taking into account that $\operatorname{ker}\left(B^{t}\right)=\{0\}$. Indeed, given $p \in P \subset L_{0}^{2}(\Omega)$ such that $B^{t} p=0$, then $(\nabla \cdot \mathbf{w}, p)=0$ for all $\mathbf{w} \in \mathbf{W}$. Now, we recall that the divergence operator $\nabla \cdot:\left(H_{0}^{1}(\Omega)\right)^{d} \rightarrow L_{0}^{2}(\Omega)$ is surjective [Tem77] and then there exists $\mathbf{w}_{p} \in H_{0}^{1}(\Omega)^{3} \subset \mathbf{W}$ such that $p=\nabla \cdot \mathbf{w}_{p}$. In consequence, $0=$ $\left(\nabla \cdot \mathbf{w}_{p}, p\right)=(p, p)$ hence $p=0$.

On the other hand, using that $\operatorname{ker} B^{t}=\{0\}$, then inequality (33) holds because it is exactly the same condition that $(I S)^{P}$, which was verified in Lemma 1.

Finally, condition $(I S)^{V}$ implies the coercivity of $a(\cdot, \cdot)$ on $\operatorname{ker}(B)$. In fact, if $\mathbf{w}_{0}=$ $\left(\mathbf{u}_{0}, v_{0}\right) \in \operatorname{ker}(B)$, then

$$
0=\left(B \mathbf{w}_{0}, p\right)=b\left(\mathbf{w}_{0}, p\right)=-\int_{\Omega}\left(\nabla_{\mathbf{x}} \cdot \mathbf{u}_{0}+\partial_{z} v_{0}\right) p=0, \quad \forall p \in P
$$

and then, using $(I S)^{V}$ (with $\beta^{V}=1$ ), we can estimate $v_{0}$ in terms of $\mathbf{u}_{0}$ :

$$
\left\|\partial_{z} v_{0}\right\|=\sup _{p \in P} \frac{\left(\partial_{z} v_{0}, p\right)}{\|p\|}=\sup _{p \in P} \frac{\left(\nabla_{\mathbf{x}} \cdot \mathbf{u}_{0}, p\right)}{\|p\|} \leq\left\|\nabla_{\mathbf{x}} \cdot \mathbf{u}_{0}\right\| \leq\left\|\nabla \mathbf{u}_{0}\right\|
$$

where the fact that $\partial_{z} v_{0} \in P$ is used in the first equality and Lemma 2 in the last inequality. Consequently, we have (32) with $\alpha_{0}=1 / 2$, because

$$
a\left(\mathbf{w}_{0}, \mathbf{w}_{0}\right)=\left\|\nabla \mathbf{u}_{0}\right\|^{2} \geq \frac{1}{2}\left(\left\|\nabla \mathbf{u}_{0}\right\|^{2}+\left\|\partial_{z} v_{0}\right\|^{2}\right)=\frac{1}{2}\left\|\mathbf{w}_{0}\right\|_{\mathbf{W}}^{2}
$$

Therefore, the existence and uniqueness of solution $(\mathbf{w}, p) \in \mathbf{W} \times P$ of (30)-(31) holds and estimates (34)-(35) yields to

$$
\|\mathbf{w}\|_{\mathbf{W}} \leq 2\|\mathbf{f}\|_{\mathbf{U}^{\prime}}, \quad\|p\|_{P} \leq \frac{3}{\beta^{P}}\|\mathbf{f}\|_{\mathbf{U}^{\prime}}
$$

Remark 4. The latter proof gives a complementary information about $(I S)^{V}$ : it can be viewed as a sufficient condition for ellipticity of the bilinear form $a(\cdot, \cdot)$ on $\operatorname{ker}(B)$. 


\section{Conforming finite elements}

Let $\mathcal{T}_{h}$ be a regular family of meshes in $\bar{\Omega}$ satisfying the usual regularity condition: there exists $\sigma>1$ such that $h_{T} \leq \sigma \rho_{T}$ for every $T \in \mathcal{T}_{h}$, where $h_{T}$ is the diameter of the triangle $T$ and $\rho_{T}$ is the maximum diameter of all circles contained in $T$ (see e.g. [Cia78]). Note that no kind of structure is assumed in $\mathcal{T}_{h}$.

Let $\mathbf{U}_{h}, V_{h}, P_{h}$ be conforming finite element spaces in $\mathbf{U}, V, P$, respectively, and let $\mathbf{X}_{h}=\mathbf{U}_{h} \times V_{h} \times P_{h}$ endowed with the $\mathbf{X}$-norm. Then, we consider the discrete hydrostatic Stokes problem: find $\boldsymbol{\chi}_{h}=\left(\mathbf{u}_{h}, v_{h}, p_{h}\right) \in \mathbf{X}_{h}$ such that

$$
\mathcal{A}\left(\chi_{h}, \bar{\chi}_{h}\right)=\mathcal{L}\left(\bar{\chi}_{h}\right) \quad \forall \bar{\chi}_{h} \in \mathbf{X}_{h},
$$

where $\mathcal{A}$ and $\mathcal{L}$ are the bilinear and linear forms defined in (22)-(23).

\subsection{Necessary and sufficient conditions for stability}

Let us to introduce the discrete inf-sup conditions

$$
\begin{array}{rll}
\sup _{0 \neq\left(\mathbf{u}_{h}, v_{h}\right) \in \mathbf{U}_{h} \times V_{h}} \frac{\left(\nabla \cdot\left(\mathbf{u}_{h}, v_{h}\right), p_{h}\right)}{\left\|\nabla \mathbf{u}_{h}, \partial_{z} v_{h}\right\|} \geq \gamma^{P}\left\|p_{h}\right\| & \forall p_{h} \in P_{h}, & (I S)_{h}^{P} \\
\sup _{0 \neq p_{h} \in P_{h}} \frac{\left(p_{h}, \partial_{z} v_{h}\right)}{\left\|p_{h}\right\|} \geq \gamma^{V}\left\|\partial_{z} v_{h}\right\| & \forall v_{h} \in V_{h}, & (I S)_{h}^{V}
\end{array}
$$

where $\gamma^{P}, \gamma^{V}>0$. We say scheme (37) has incompressible stability if $\gamma^{P}$ is independent of $h$, and "hydrostatic" stability if $\gamma^{V}$ is independent of $h$.

Theorem 6. The following statements are equivalent:

1. $\mathbf{U}_{h}, V_{h}$ and $P_{h}$ verify conditions $(I S)_{h}^{P}$ and $(I S)_{h}^{V}$.

2. Scheme (37) is well-posed in $\mathbf{X}_{h}$.

In this case, there exists an unique solution $\chi_{h}=\left(\mathbf{u}_{h}, v_{h}, p_{h}\right) \in \mathbf{X}_{h}$ of (37) and the following estimates hold:

$$
\left\|\nabla \mathbf{u}_{h}\right\| \leq\|\mathbf{f}\|_{\mathbf{U}^{\prime}}, \quad\left\|\partial_{z} v_{h}\right\| \leq \frac{1}{\gamma^{V}}\|\mathbf{f}\|_{\mathbf{U}^{\prime}}, \quad\left\|p_{h}\right\| \leq \frac{2}{\gamma^{P}}\|\mathbf{f}\|_{\mathbf{U}^{\prime}}
$$

Proof. If $(I S)_{h}^{P}$ and $(I S)_{h}^{V}$ hold, it is enough to repeat the proof of Theorem 3, replacing the spaces $\mathbf{U}, V$ and $P$ by $\mathbf{U}_{h}, V_{h}$ and $P_{h}$, to get

$$
\sup _{0 \neq \overline{\boldsymbol{\chi}} \in \mathbf{X}_{h}} \frac{\mathcal{A}\left(\chi_{h}, \bar{\chi}_{h}\right)}{\left\|\bar{\chi}_{h}\right\|_{\mathbf{X}}} \geq \gamma\left\|\chi_{h}\right\|_{\mathbf{X}}, \quad \forall \chi_{h} \in \mathbf{X}_{h},
$$

for a certain $\gamma=\gamma\left(\gamma^{P}, \gamma^{V}\right)$ (independent of $h$ if $\gamma^{P}$ and $\gamma^{V}$ are independent of $h$ ), which is the discrete counterpart of (25). Moreover, since $\mathcal{A}$ is symmetric, this condition is equivalent to the existence of solution, uniqueness and energy estimates (see, for example, [EG04]). Finally, specific stability estimates (38) can be obtained directly as in proof of Theorem 3. 
Remark 5. As in the continuous case, if we denote $\mathbf{W}_{h}=\mathbf{U}_{h} \times V_{h}$, the discrete problem (37) is equivalent to the mixed formulation:

$$
\begin{aligned}
a\left(\mathbf{w}_{h}, \overline{\mathbf{w}}_{h}\right)+b\left(\overline{\mathbf{w}}_{h}, p\right) & =\left\langle f, \overline{\mathbf{u}}_{h}\right\rangle \quad \forall \overline{\mathbf{w}}_{h} \in \mathbf{W}_{h}, \\
b\left(\mathbf{w}_{h}, \bar{p}\right) & =0 \quad \forall \bar{p} \in P_{h},
\end{aligned}
$$

where $a(\cdot, \cdot)$ and $b(\cdot, \cdot)$ are defined as in Section 4 . Now $B_{h}: \mathbf{W}_{h} \rightarrow P_{h}^{\prime}$ and $B_{h}^{t}: P_{h} \rightarrow \mathbf{W}_{h}^{\prime}$. Consequently, condition $(I S)_{h}^{V}$ is related again with elipticity of $a(\cdot, \cdot)$ on $\operatorname{ker}\left(B_{h}\right)$.

Corollary $\mathbf{7}$ (Error estimates). Let $(\mathbf{u}, v, p)$ and $\left(\mathbf{u}_{h}, v_{h}, p_{h}\right)$ be the solutions of (30)-(31) and (40)-(41), respectively. There is a constant $C>0$ (depending on constants $\gamma^{P}$ and $\gamma^{V}$ of $(I S)_{h}^{P}$ and $\left.(I S)_{h}^{V}\right)$ such that:

$$
\begin{aligned}
\| \nabla\left(\mathbf{u}-\mathbf{u}_{h}\right), \partial_{z}(v- & \left.v_{h}\right), p-p_{h} \| \\
& \leq C\left(\inf _{\overline{\mathbf{u}}_{h} \in \mathbf{U}_{h}}\left\|\nabla\left(\mathbf{u}-\overline{\mathbf{u}}_{h}\right)\right\|+\inf _{\bar{v}_{h} \in V_{h}}\left\|\partial_{z}\left(v-\bar{v}_{h}\right)\right\|+\inf _{\bar{p}_{h} \in P_{h}}\left\|p-\bar{p}_{h}\right\|\right) .
\end{aligned}
$$

Proof. It suffices to apply the Céa's lemma in the Banach-Necas-Babuska framework, which provides error estimates for generalized elliptic problems like (37) (see for instance [EG04, Lemma 2.28]).

More precise error estimates could be provided in order to obtain an optimal expression of the constant $C$ as a function of $\gamma^{P}$ and $\gamma^{V}$.

\subsection{Sufficient conditions for "incompressible" stability}

Lemma 8 (Sufficient condition for $\left.(I S)_{h}^{P}\right)$. Let $\mathbf{X}_{h}=\mathbf{U}_{h} \times V_{h} \times P_{h}$. Assume that exist $\mathbf{U}_{h}^{S} \subset \mathbf{U}_{h} \cap H_{0}^{1}(\Omega)^{2}$ and $V_{h}^{S} \subset V_{h} \cap H_{0}^{1}(\Omega)$ such that the combination $\left(\mathbf{U}_{h}^{S}, V_{h}^{S}\right)-P_{h}$ is Stokes-stable, namely: there exists $C>0$ such that

$$
\sup _{0 \neq\left(\mathbf{u}_{h}, v_{h}\right) \in \mathbf{U}_{h}^{S} \times V_{h}^{S}} \frac{\left(\nabla \cdot\left(\mathbf{u}_{h}, v_{h}\right), p_{h}\right)}{\left\|\nabla \mathbf{u}_{h}, \nabla v_{h}\right\|} \geq C\left\|p_{h}\right\| \quad \forall p \in P_{h} .
$$

Then $\mathbf{X}_{h}$ verifies $(I S)_{h}^{P}$.

Proof. Analogously to the first part of Lemma 1.

Remark 6. The reciprocal implication of Lemma 8 is an open problem. Namely it is not clear if $(I S)_{h}^{P}$ implies $(I S)_{h}$ for some $\mathbf{U}_{h}^{S} \times V_{h}^{S} \subset\left(\mathbf{U}_{h} \times V_{h}\right) \cap H_{0}^{1}(\Omega)^{d}$.

\subsection{Necessary conditions for stability}

Now, we give some necessary conditions obtained from the algebraic version of the scheme.

Lemma 9. Let $N_{u}=\operatorname{dim} \mathbf{U}_{h}, N_{v}=\operatorname{dim} V_{h}$ and $N_{p}=\operatorname{dim} P_{h}$.

1. If $(I S)_{h}^{P}$ holds then $N_{p} \leq N_{u}+N_{v}$.

2. If $(I S)_{h}^{V}$ holds then $N_{v} \leq N_{p}$. 
Proof. 1. As in the Stokes case, given a fixed mesh $\mathcal{T}_{h}$, a necessary condition for $(I S)_{h}^{P}$ (with a constant $\gamma^{P}>0$ dependent on $h$ ) is $\operatorname{ker}\left(B_{h}^{t}\right)=\{0\}$. Indeed $(I S)_{h}^{P}$ implies that if $B_{h}^{t} p_{h}=0$ in $\mathbf{W}_{h}^{\prime}$, namely $\left(B_{h}^{t} p_{h}, \mathbf{w}_{h}\right)=0$ for all $\mathbf{w}_{h} \in \mathbf{W}_{h}$, then $p_{h}=0$.

Scheme (37) is equivalent to the algebraic system: find $(W, P) \in \mathbb{R}^{N_{u}+N_{v}} \times \mathbb{R}^{N_{p}}$, such that

$$
\left(\begin{array}{cc}
A & B^{t} \\
B & 0
\end{array}\right)\left(\begin{array}{c}
W \\
P
\end{array}\right)=\left(\begin{array}{c}
F \\
0
\end{array}\right)
$$

Here $A$ denotes the square matrix of order $N_{w}=N_{u}+N_{v}$ whose elements are $a_{i j}=$ $a\left(\Phi_{i}, \Phi_{j}\right)$, where $\Phi_{i}=\left(\varphi_{i}^{\mathbf{u}}, 0\right), i=1, \ldots, N_{u}$ and $\Phi_{N_{u}+i}=\left(0, \varphi_{i}^{v}\right), i=1, \ldots, N_{v}$ (and $\left\{\varphi_{i}^{\mathbf{u}}\right\},\left\{\varphi_{i}^{v}\right\}$ are a basis, respectively, of $\mathbf{U}_{h}$ and $\left.V_{h}\right)$, thus $\left\{\Phi_{i}, i=1, \ldots, N_{w}\right\}$ is a basis of $\mathbf{W}_{h}=\mathbf{U}_{h} \times V_{h}$. On the other hand, $B$ is the matrix of order $N_{p} \times N_{w}$ whose elements are $b_{i j}=b\left(\psi_{i}, \Phi_{j}\right)$, where $\psi_{i}, i=1, \ldots, N_{p}$, is a basis of $P_{h}$.

If $(W, P)$ is a solution of (42) then $B^{t} P=F-A W$ and consequently the uniqueness of $P$ is equivalent to $\operatorname{ker}\left(B^{t}\right)=0$ (where now $B$ is the matrix defined above). In particular, $B^{t}$ can be considered as a linear form between two finite-dimensional spaces, $B^{t}: \mathbb{R}^{N_{p}} \rightarrow \mathbb{R}^{N_{w}}$, and consequently $\operatorname{dim}\left(\operatorname{ker}\left(B^{t}\right)\right)+\operatorname{dim}\left(\operatorname{Img}\left(B^{t}\right)\right)=N_{p}$. Then, if $(I S)_{h}^{P}$ holds, $\operatorname{ker}\left(B^{t}\right)=0$, and consequently $N_{p}=\operatorname{dim}\left(\operatorname{Img}\left(B^{t}\right)\right) \leq N_{w}$. Thus $N_{p} \leq N_{w}=N_{u}+N_{v}$ is a necessary condition for $(I S)_{h}^{P}$.

2. Now we will go beyond the Stokes system, exploiting the peculiarities of the hydrostatic problem. The matrix $A$ is specifically of the form

$$
A=\left(\begin{array}{cc}
A_{1} & 0 \\
0 & 0
\end{array}\right)
$$

where $A_{1}$ is a $N_{u} \times N_{u}$ matrix whose elements are $\left(\nabla \varphi_{i}^{\mathbf{u}}, \nabla \varphi_{j}^{\mathbf{u}}\right)$. Similarly, $F=\left(F_{1}, 0\right)^{t}$ and the linear system (42) is written as:

$$
\left(\begin{array}{ccc}
A_{1} & 0 & B_{1}^{t} \\
0 & 0 & B_{2}^{t} \\
-B_{1} & -B_{2} & 0
\end{array}\right)\left(\begin{array}{l}
U \\
V \\
P
\end{array}\right)=\left(\begin{array}{c}
F_{1} \\
0 \\
0
\end{array}\right),
$$

where $\left(B_{1}\right)_{i j}=\left(\nabla_{\mathbf{x}} \cdot \varphi_{j}^{\mathbf{u}}, \psi_{i}\right)$ and $\left(B_{2}\right)_{i j}=\left(\partial_{z} \varphi_{j}^{v}, \psi_{i}\right)$.

Since $V$ is defined only by the system $B_{2} V=-B_{1} U$, uniqueness of $V$ is equivalent to $\operatorname{ker}\left(B_{2}\right)=\{0\}$. But $B_{2}$ can be considered as a linear application $B_{2}: \mathbb{R}^{N_{v}} \rightarrow \mathbb{R}^{N_{p}}$ and then $\operatorname{dim}\left(\operatorname{ker}\left(B_{2}\right)\right)+\operatorname{dim}\left(\operatorname{Img}\left(B_{2}\right)\right)=N_{v}$. Thus, if $(I S)_{h}^{V}$ holds, $\operatorname{dim}\left(\operatorname{Img}\left(B_{2}\right)\right)=N_{v}$. Finally, since $\operatorname{Img}\left(B_{2}\right) \subset \mathbb{R}^{N_{p}}, N_{v} \leq N_{p}$.

\section{Applications and first numerical results}

\subsection{Unstable approximations of the Stokes problem with vanishing ver- tical viscosity}

It is well known (see e.g. [BF91]) that well-posedness of the finite element approximations of the Stokes problem depends on choosing finite element spaces verifying the discrete inf-sup condition $(I S)_{h}$. This assertion remains true in the case of the anisotropic Stokes 
equations related to (5)-(7),

$$
\begin{aligned}
-\Delta_{\nu} u+\nabla_{\mathbf{x}} p=\mathbf{f} & \text { in } \Omega, \\
-\varepsilon^{2} \Delta_{\nu} v+\partial_{z} p=g & \text { in } \Omega, \\
\nabla_{\mathbf{x}} \cdot u+\partial_{z} v=0 & \text { in } \Omega,
\end{aligned}
$$

endowed with boundary conditions (8)-(10). But when $\varepsilon \rightarrow 0$, this system tends to the hydrostatic Stokes problem (1)-(3) (see [AG01] for the nonlinear unsteady case and [BL92] for the steady case). According to Theorem 6, when a finite element approximation is made for the limit problem (1)-(3), its stability requires a choice of finite element spaces verifying not only the condition $(I S)_{h}^{P}$, which is related to $(I S)_{h}$, but also the constraint $(I S)_{h}^{V}$, which is a new restriction, not required in the standard Stokes problem.

Hence, one can expect ill-behavior of (44)-(46) when $\varepsilon \rightarrow 0$ for some of the standard Stokes-stable FE approximations, like Taylor-Hood (which is denoted in this work by $\left.\left(\mathcal{P}_{2}, \mathcal{P}_{2}\right)-\mathcal{P}_{1}\right)$ and $\mathcal{P}_{1}$-bubble elements (denoted by $\left.\left(\mathcal{P}_{1, b}, \mathcal{P}_{1, b}\right)-\mathcal{P}_{1}\right)$. This expectation will be confirmed in Section 6.2 for the limit case $\varepsilon=0$, both numerically and analytically. We present before some numerical tests showing the ill-behavior of standard Stokes-stable finite elements approximations applied to the anisotropic Stokes problem when $\varepsilon>0$ is small enough.

Test 1 (Anisotropic Stokes cavity test when $\varepsilon \rightarrow 0$ ). We have considered an unstructured mesh with 3.792 triangles in the square $(0,1) \times(-1,0) \subset \mathbb{R}^{2}$ and finite element approximations of the anisotropic Stokes problem (44)-(46) with boundary conditions (8)-(10), $\mathbf{f}=0, g=0$ and $\mathbf{g}_{s}=1$.

The scheme has been slightly altered by introducing a standard pressure penalization technique, transforming divergence equation (46) in

$$
\left(\partial_{x} u+\partial_{y} v, \bar{p}\right)+\delta(p, \bar{p})=0 \quad \forall \bar{p} \in P_{h},
$$

where $\delta$ is a small penalization parameter (in this example, $\delta=10^{-10}$ ). With this approximation, the condition $\int_{\Omega} p=0$ is satisfied implicitly and it is no longer necessary to include it in the definition of $P_{h}$, taking $P_{h}=\left\{p_{h} \in C^{0}(\bar{\Omega}) /\left.p\right|_{T} \in \mathcal{P}_{1}, \quad \forall T \in \mathcal{T}_{h}\right\}$.

Note that this penalization technique is widely used in FE implementations of Stokes mixed problem (see e.g. examples in [PHLHM], where $\delta=10^{-10}$ and $\delta=10^{-8}$ are chosen). We have just translated it to the "quasi-hydrostatic" case $\left(\varepsilon \leq 10^{-3}\right)$. Note that $\delta$ must not be either "too high" (for avoiding the introduction of numerical viscosity) or "too small" (for vanishing the mean of $p$ in $\Omega$ ). After doing numerical experiments for different values of $\delta \in\left(10^{-20}, 10^{-1}\right)$, where the mean of $p$ in $\Omega$ (i.e. $\int_{\Omega} p / \int_{\Omega} 1$ ) and other residual indicators are tested (namely incompressibility $\|\nabla \cdot(u, v)\|_{L^{2}}$ and hydrostatic $\left\|\partial_{z} p-g\right\|_{L^{2}}$ restrictions) we confirm that $\delta \simeq 10^{-10}$ is adequate, obtaining a small mean of $p$ (for instance $2.56815 \cdot 10^{-7}$ for $\varepsilon=10^{-3}$ and similar values for other choices of $\varepsilon$ ) while the rest of indicators are not significantly affected with respect to the not penalized case $(\delta=0)$.

The experiments have been programmed using the FreeFem ++ software [PHLHM]. Velocity streamlines obtained for both $\left(\mathcal{P}_{2}, \mathcal{P}_{2}\right)-\mathcal{P}_{1}$ and $\left(\mathcal{P}_{1, b}, \mathcal{P}_{1, b}\right)-\mathcal{P}_{1}$ finite elements are shown, respectively, in Figures 2 and 3 , each one for $\varepsilon=10^{-2}, 10^{-3}$ and $10^{-4}$. In both cases one can see that the results are correct for $\varepsilon=10^{-2}$, but the velocity field start 


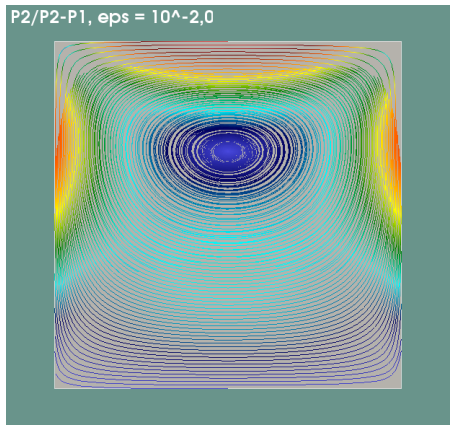

(a) $\varepsilon=10^{-2}$

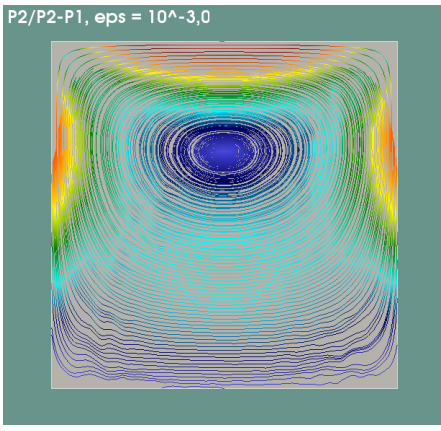

(b) $\varepsilon=10^{-3}$

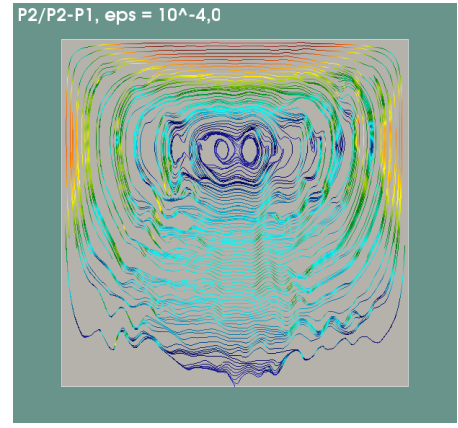

(c) $\varepsilon=10^{-4}$

Figure 2: Velocity streamlines for $\left(\mathcal{P}_{2}, \mathcal{P}_{2}\right)-\mathcal{P}_{1}$ when $\varepsilon \rightarrow 0$

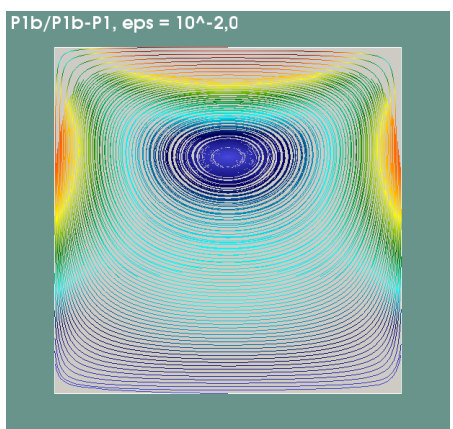

(a) $\varepsilon=10^{-2}$

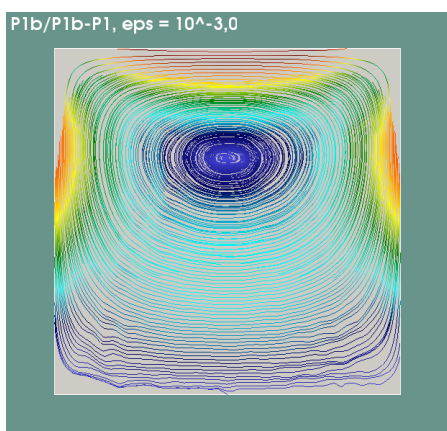

(b) $\varepsilon=10^{-3}$

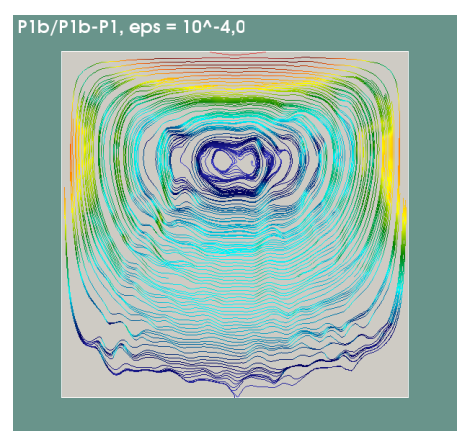

(c) $\varepsilon=10^{-4}$

Figure 3: Velocity streamlines for $\left(\mathcal{P}_{1, b}, \mathcal{P}_{1, b}\right)-\mathcal{P}_{1}$ when $\varepsilon \rightarrow 0$ 


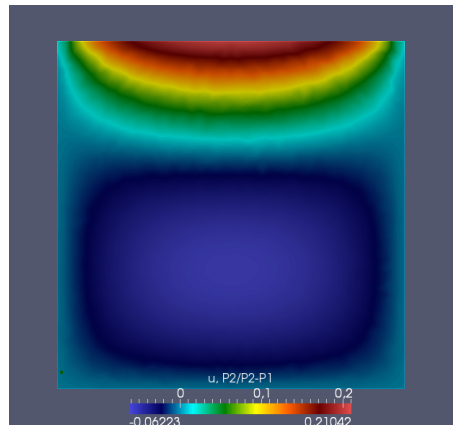

(a) $u_{h}$

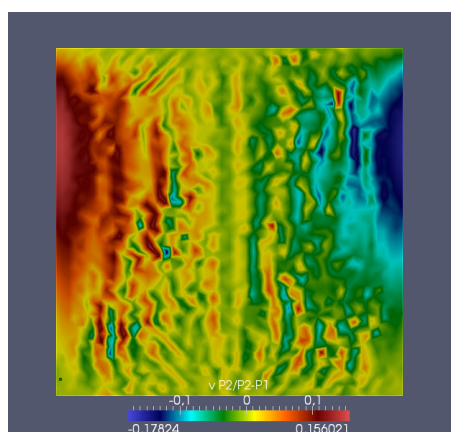

(b) $v_{h}$

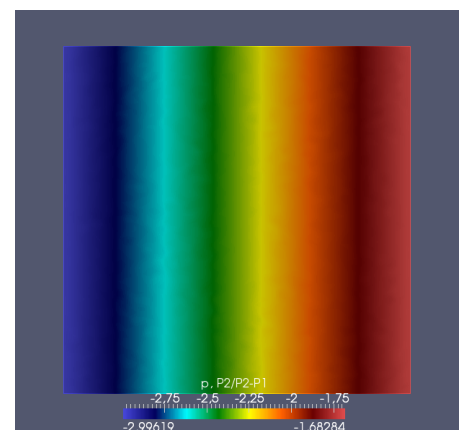

(c) $p_{h}$

Figure 4: Vertical velocity instability for $\left(\mathcal{P}_{2}, \mathcal{P}_{2}\right)-\mathcal{P}_{1}$ and $\varepsilon=10^{-4}$.

presenting small instabilities for $\varepsilon=10^{-3}$ and big oscillations for $\varepsilon=10^{-4}$. Recall that values $\varepsilon=10^{-3}$ and $\varepsilon=10^{-4}$ are standard scales in Oceanography (Section 2).

Figure 4 shows isolines of $u_{h}, v_{h}$ and $p_{h}$ for $\left(\mathcal{P}_{2}, \mathcal{P}_{2}\right)-\mathcal{P}_{1}$ and $\varepsilon=10^{-4}$, where oscillations are only appreciated for the vertical velocity (Figure $4 \mathrm{~b})$. This suggest that the reason for instabilities of the velocity field (in figure $2 c$ ) is the fact that $\left(\mathcal{P}_{2}, \mathcal{P}_{2}\right)-\mathcal{P}_{1}$ does not verify an inf-sup condition bounding $v_{h}$ like $(I S)_{h}^{V}$. The results for $\left(\mathcal{P}_{1, b}, \mathcal{P}_{1, b}\right)-\mathcal{P}_{1}$ are analogue.

\subsection{Unstable approximations of the hydrostatic Stokes problem}

Here we will show that some standard Stokes-stable finite element spaces lose their stability when $\varepsilon=0$ is taken in the vertical momentum equation (45).

Let us consider the hydrostatic Stokes equations (1)-(3) with boundary conditions (8)(9). First, $u_{h}, v_{h}, p_{h}$ will be approximated by $\left(\mathcal{P}_{2}, \mathcal{P}_{2}\right)-\mathcal{P}_{1}$ elements and, using the analytic results obtained in Section 5, we will prove that this combination is not hydrostaticstable. A numerical experiment confirming this fact will be shown. Finally, this theoretical analysis will be extended to $\left(\mathcal{P}_{2}, \mathcal{P}_{2}\right)-\mathcal{P}_{0}$ and $\left(\mathcal{P}_{1, b}, \mathcal{P}_{1, b}\right)-\mathcal{P}_{1}$.

The $\left(\mathcal{P}_{2}, \mathcal{P}_{2}\right)-\mathcal{P}_{1}$ case. Let

$$
\begin{aligned}
& U_{h}=\left\{u_{h} \in H_{b, l}^{1} \cap C^{0}(\bar{\Omega}) /\left.u\right|_{T} \in \mathcal{P}_{2}, \quad \forall T \in \mathcal{T}_{h}\right\}, \\
& V_{h}=\left\{v_{h} \in H_{z, 0}^{1}(\Omega) \cap C^{0}(\bar{\Omega}) /\left.v\right|_{T} \in \mathcal{P}_{2}, \quad \forall T \in \mathcal{T}_{h}\right\}, \\
& P_{h}=\left\{p_{h} \in L_{0}^{2}(\Omega) \cap C^{0}(\bar{\Omega}) /\left.p\right|_{T} \in \mathcal{P}_{1}, \quad \forall T \in \mathcal{T}_{h}\right\} .
\end{aligned}
$$

We will show that the second necessary condition given in Lemma 9 does not hold and then $(I S)_{h}^{V}$ is not verified. Note that Theorem 6 states that stability of the discrete hydrostatic Stokes problem is equivalent to both $(I S)_{h}^{P}$ and $(I S)_{h}^{V}$ are verified.

Let us consider, for example, the structured mesh shown in Figure 5. The DOF for $u_{h}$ are $q_{0}, x_{1}, \ldots, x_{8}, y_{3}, y_{4}$ and $q_{2}$ (because $u_{h}$ has not got restrictions on $\Gamma_{s}$ ), then $N_{u}=12$. On the other hand, since $v_{h}$ has not got restrictions on $\Gamma_{l}$, then $N_{v}=15$ (the DOF for $v_{h}$ are $q_{0}, x_{1}, \ldots, x_{8}, y_{1}, y_{2}, y_{5}, y_{6}$ and $\left.q_{4}, q_{8}\right)$. Finally, the DOF for $p_{h}$ are only $x_{0}, q_{1}, \ldots, q_{8}$, 


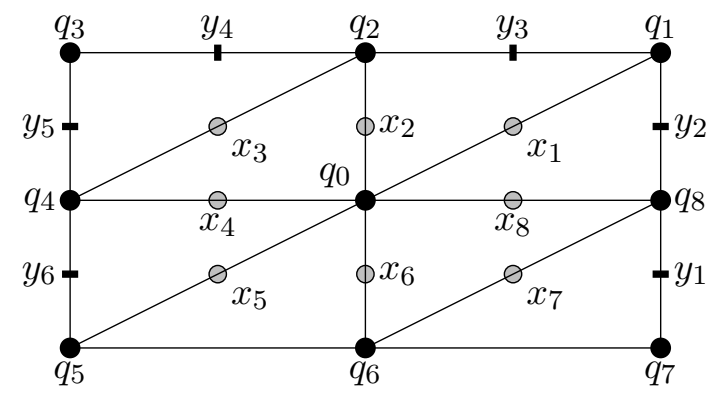

Figure 5: Degrees of freedom $\left(\mathcal{P}_{2}, \mathcal{P}_{2}\right)-\mathcal{P}_{1}$ in a $2 \times 2$ structured mesh

then $N_{p}=9$ (in fact, $N_{p}=8$ considering the constraint $\int_{\Omega} p_{h}=0$ ). Then $N_{p}<N_{v}$ and Lemma 9 says that $(I S)_{h}^{V}$ does not hold.

This fact can be generalized to an analogous structured mesh $\mathcal{T}_{h}$ defined by $n \times n$ subintervals, with $n \in \mathbb{N}, n>2$. Then the number of vertices is $(n+1)^{2}$, of which $(n-1)^{2}$ are in the interior of $\Omega$. The number of midpoints is $n(3 n+2)$, of which $2 n$ are in $\Gamma_{S} \cup \Gamma_{b}$ and $2 n$ in $\Gamma_{l}$. As consequence, $N_{u}=(n-1)^{2}+(n-1)+n(3 n+2)-3 n=4 n^{2}-2 n$, $N_{v}=(n-1)^{2}+2(n-1)+n(3 n+2)-2 n=4 n^{2}$, and, taking into account the null mean restriction of functions in $P_{h}, N_{p}=(n+1)^{2}-1=n^{2}+2 n$. Therefore $N_{p}<N_{v}$ and $(I S)_{h}^{V}$ does not hold. Then, from Theorem 6 , approximation $\left(\mathcal{P}_{2}, \mathcal{P}_{2}\right)-\mathcal{P}_{1}$ for $u_{h}, v_{h}, p_{h}$ is not hydrostatic Stokes-stable.

On the other hand, defining $U_{h}^{S}=U_{h} \cap H_{0}^{1}(\Omega)$ and $V_{h}^{S}=V_{h} \cap H_{0}^{1}(\Omega)$, since combination $\left(U_{h}, V_{h}^{S}\right)-P_{h}$ is Stokes-stable, then, by using Lemma $8,(I S)_{h}^{P}$ holds.

In order to check numerically the predictions of the numerical analysis, let us show some computational simulations.

Test 2 (Hydrostatic Stokes, $\left(\mathcal{P}_{2}, \mathcal{P}_{2}\right)-\mathcal{P}_{1}$ and $\left(\mathcal{P}_{1, b}, \mathcal{P}_{1, b}\right)-\mathcal{P}_{1}$, cavity test). We discretize the domain of Test 1 , with $h \simeq 1 / 40$, by both structured and unstructured meshes, approaching hydrostatic Stokes scheme (1)-(2)-(3)-(8)-(9) using finite element spaces $U_{h}$, $V_{h}, P_{h}$ given in (48)-(50). As in Test 1 (Section 6.1) we set $\mathbf{f}=0, g=0, \mathbf{g}_{s}=1$ and pressure penalization in the divergence equation (47), with parameter $\delta=10^{-10}$.

The graphics obtained for velocity and pressure in the hydrostatic case, $\varepsilon=0$, are indistinguishable from the ones obtained in Test 1 for $\varepsilon=10^{-4}$ (Figure 4). In particular, oscillations for the vertical velocity $v_{h}$ are evident, but not for $u_{h}$ or $p_{h}$. These results agree with the analysis made in this paper, since we have proven that $\left(\mathcal{P}_{2}, \mathcal{P}_{2}\right)-\mathcal{P}_{1}$ does not verify $(I S)_{h}^{V}$, and then we cannot hope stability for $v_{h}$. On the other hand, the pressure does not present oscillations, which it can be expected because $(I S)_{h}^{P}$ holds.

The cases $\left(\mathcal{P}_{2}, \mathcal{P}_{2}\right)-\mathcal{P}_{0}$ and $\left(\mathcal{P}_{1, b}, \mathcal{P}_{1, b}\right)-\mathcal{P}_{1} \quad$ are analogous to $\left(\mathcal{P}_{2}, \mathcal{P}_{2}\right)-\mathcal{P}_{1}$ : they both verify $(I S)_{h}^{P}$, because they are stable-Stokes, but none of them verify $(I S)_{h}^{V}$, because the space for vertical velocities is "too rich" with respect to the pressure space.

For example, for $\left(\mathcal{P}_{2}, \mathcal{P}_{2}\right)-\mathcal{P}_{0}$, since

$$
P_{h}=\left\{p_{h} \in L_{0}^{2}(\Omega) /\left.p\right|_{T} \in \mathcal{P}_{0}, \quad \forall T \in \mathcal{T}_{h}\right\},
$$

the number of DOF for pressure in a structured mesh defined by $n \times n$ sub-intervals 
(similar to Figure 5) is $N_{p}=2 n^{2}-1$. But we have already seen that $N_{v}=4 n^{2}$, therefore $N_{p}<N_{v}$ and $(I S)_{h}^{V}$ does not hold.

With regard to $\left(\mathcal{P}_{1, b}, \mathcal{P}_{1, b}\right)-\mathcal{P}_{1}$, if we take

$$
\begin{aligned}
U_{h} & =\left\{u_{h} \in H_{b, l}^{1} \cap C^{0}(\bar{\Omega}) /\left.u_{h}\right|_{T} \in \mathcal{P}_{1, b}, \quad \forall T \in \mathcal{T}_{h}\right\}, \\
V_{h} & =\left\{v_{h} \in H_{z, 0}^{1}(\Omega) \cap C^{0}(\bar{\Omega}) /\left.v_{h}\right|_{T} \in \mathcal{P}_{1, b}, \quad \forall T \in \mathcal{T}_{h}\right\},
\end{aligned}
$$

then $N_{v}=(n-1)^{2}+2(n-1)+2 n^{2}=3 n^{2}$. But we have already seen that $N_{p}=n^{2}+2 n$, consequently $N_{p}<N_{v}$ therefore $\left(\mathcal{P}_{1, b}, \mathcal{P}_{1, b}\right)-\mathcal{P}_{1}$ is not hydrostatic Stokes-stable.

The numerical tests using $\left(\mathcal{P}_{1, b}, \mathcal{P}_{1, b}\right)-\mathcal{P}_{1}$ and $\left(\mathcal{P}_{2}, \mathcal{P}_{2}\right)-\mathcal{P}_{0}$ are analogous to the case $\left(\mathcal{P}_{2}, \mathcal{P}_{2}\right)-\mathcal{P}_{1}$, presenting spurious oscillations for the vertical velocity, which agree with the numerical analysis.

\subsection{Stability of $\left(\mathcal{P}_{2}, \mathcal{P}_{1}\right)-\mathcal{P}_{0}$ for the hydrostatic Stokes problem}

Let $\mathbf{X}_{h}=U_{h} \times V_{h} \times P_{h}$, with $U_{h}$ defined in (48), $p_{h}$ in (51) and

$$
V_{h}=\left\{v_{h} \in H_{z, 0}^{1}(\Omega) \cap C^{0}(\bar{\Omega}) /\left.v\right|_{T} \in \mathcal{P}_{1}, \quad \forall T \in \mathcal{T}_{h}\right\},
$$

Theorem 10. $\left(\mathcal{P}_{2}, \mathcal{P}_{1}\right)-\mathcal{P}_{0}$ satisfies $(I S)_{h}^{P}$ and $(I S)_{h}^{V}$.

Proof. $(I S)_{h}^{P}$ follows from Lemma 8 because $\left(\mathcal{P}_{2}, \mathcal{P}_{1}\right)-\mathcal{P}_{0}$ is Stokes-stable, see [Ste90] for a proof based on the Stenberg's macroelement technique.

$(I S)_{h}^{V}$ can be easily verified, by means of a similar argument to the second part of the proof of Lemma 1. Indeed, for each $v_{h} \in V_{h}$, we consider $\widetilde{p}_{h}=\partial_{z} v_{h} \in L_{0}^{2}(\Omega)$. Then $\left.\widetilde{p}_{h}\right|_{T} \in \mathcal{P}_{0}$ for each $T \in \mathcal{T}_{h}$ and,

$$
\int_{\Omega} \widetilde{p}_{h}=\int_{S} \int_{-D(x)}^{0} \partial_{z} v_{h}(x, z) d z d x=\int_{S}\left(v_{h}(x, 0)-v_{h}(x,-D(x))\right) d x=0
$$

hence, $\widetilde{p}_{h} \in P_{h}$ and of course $\left(\widetilde{p}_{h}, \partial_{z} v_{h}\right)=\left\|\partial_{z} v_{h}\right\|^{2}$ and $\left\|\widetilde{p}_{h}\right\|=\left\|\partial_{z} v_{h}\right\|$. Consequently $(I S)_{h}^{V}$ holds with $\gamma^{V}=1$ and then $\mathbf{X}_{h}$ is hydrostatic-stable.

Corollary 11 (Error estimates and $\left.\left(\mathcal{P}_{2}, \mathcal{P}_{1}\right)-\mathcal{P}_{0}\right)$. If the exact solution $(u, v, p) \in H^{2}(\Omega) \times$ $H^{2}(\Omega) \times H^{1}(\Omega)$, then there is a constant $C>0$ such that

$$
\left\|\nabla\left(u-u_{h}\right), \partial_{z}\left(v-v_{h}\right), p-p_{h}\right\| \leq C h\|u, v, p\|_{H^{2} \times H^{2} \times H^{1}} .
$$

Proof. It is a direct consequence of Corollary 7.

Remark 7. It is clear (see the proof of Theorem 10) that $\left(\mathcal{P}_{1}, \mathcal{P}_{1}\right)-\mathcal{P}_{0}$ verifies $(I S)_{h}^{V}$. But, on the other hand, it is well known that $\left(\mathcal{P}_{1}, \mathcal{P}_{1}\right)-\mathcal{P}_{0}$ is not Stokes-stable in general meshes. Using that, it can be proved that $\left(\mathcal{P}_{1, b}, \mathcal{P}_{1}\right)-\mathcal{P}_{0}$ is not Stokes-stable [GR12]. Although it is not clear (see Remark 6) if $(I S)_{h}^{P}$ holds or not, our numerical experiments suggest that $(I S)_{h}^{P}$ is not verified (see Test 3 ). Therefore, it seems rather probable that both $\left(\mathcal{P}_{1}, \mathcal{P}_{1}\right)-\mathcal{P}_{0}$ and $\left(\mathcal{P}_{1, b}, \mathcal{P}_{1}\right)-\mathcal{P}_{0}$ are not hydrostatic-stable. In this sense, $\left(\mathcal{P}_{2}, \mathcal{P}_{1}\right)-\mathcal{P}_{0}$ can be qualified as a "minimal" stable finite element combination with $\mathcal{P}_{0}$ pressures, both for Stokes and hydrostatic Stokes problems. 


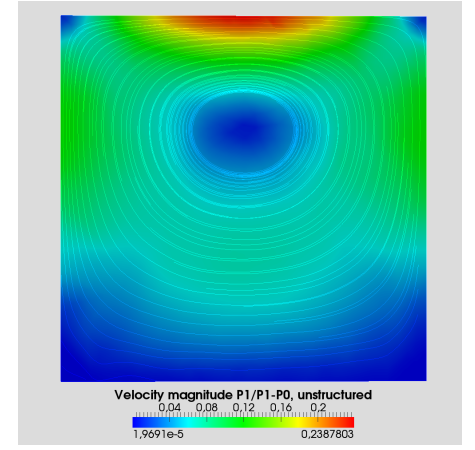

(a) Velocity magnitude and streamlines

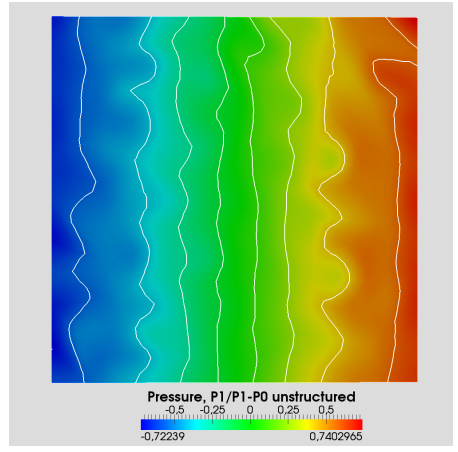

(b) Pressure surface plot and isolines

Figure 6: Velocity and pressure for $\left(\mathcal{P}_{1}, \mathcal{P}_{1}\right)-\mathcal{P}_{0}$ hydrostatic Stokes

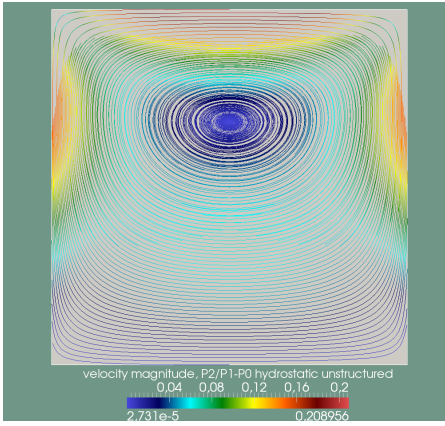

(a) Velocity streamlines

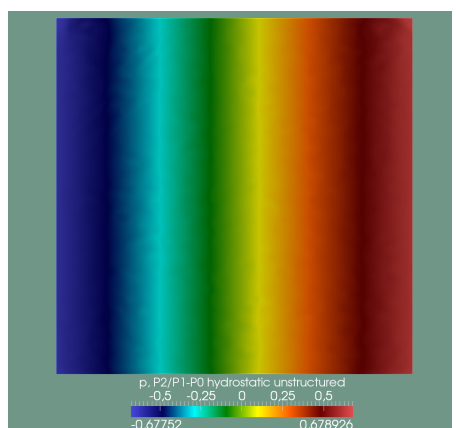

(b) Pressure

Figure 7: Velocity and pressure for $\left(\mathcal{P}_{2}, \mathcal{P}_{1}\right)-\mathcal{P}_{0}$ hydrostatic Stokes

\section{Numerical tests}

Test $3\left(\left(\mathcal{P}_{1}, \mathcal{P}_{1}\right)-\mathcal{P}_{0}\right.$ cavity test $)$. We adapt Test 2 to $\left(\mathcal{P}_{1}, \mathcal{P}_{1}\right)-\mathcal{P}_{0}$. As commented in Remark \%, $\left(\mathcal{P}_{1}, \mathcal{P}_{1}\right)-\mathcal{P}_{0}$ verifies $(I S)_{h}^{V}$ but not the discrete Stokes inf-sup condition $(I S)_{h}$. According to our numerical tests, we conjecture that $(I S)_{h}^{P}$ is not verified too. For example, Figure 6 shows the results for $\left(\mathcal{P}_{1}, \mathcal{P}_{1}\right)-\mathcal{P}_{0}$, where spurious oscillations for the pressure appears. By the contrary, velocity streamlines (Figure 6a) seem correct, what is coherent with the fact that $(I S)_{h}^{V}$ holds.

Test $4\left(\left(\mathcal{P}_{2}, \mathcal{P}_{1}\right)-\mathcal{P}_{0}\right.$ cavity test $)$. We adapt Test 2 to $\left(\mathcal{P}_{2}, \mathcal{P}_{1}\right)-\mathcal{P}_{0}$. Velocity streamlines and pressure contours have been plotted in Figure $\%$. Results are acceptable, presenting no spurious oscillations, what is coherent with previous theoretical analysis. Note that, although a relatively coarse mesh $(h \simeq 1 / 40)$ was employed, the qualitative behavior of flow circulation and the hydrostatic pressure agree with results obtained in literature (using integro-differential schemes in structured meshes, see e.g. [CGS12, GR05, Kim13]).

Test 5 (Approximation of accuraty orders for $\left.\left(\mathcal{P}_{2}, \mathcal{P}_{1}\right)-\mathcal{P}_{0}\right)$. We have considered the 


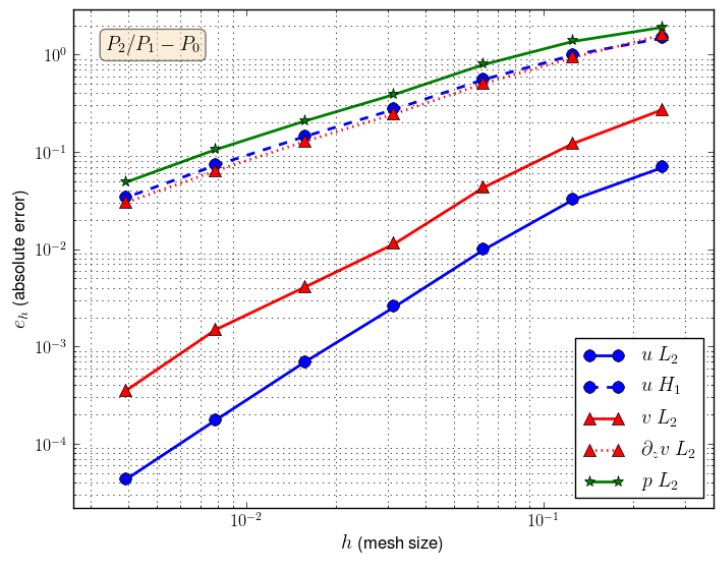

Figure 8: Velocity and pressure errors for $\left(\mathcal{P}_{2}, \mathcal{P}_{1}\right)-\mathcal{P}_{0}$ hydrostatic Stokes

\begin{tabular}{lrrrrrrr}
\hline$h_{2}$ & & $2^{-3}$ & $2^{-4}$ & $2^{-5}$ & $2^{-6}$ & $2^{-7}$ & $2^{-8}$ \\
\hline \multirow{2}{*}{$u$} & $\left\|u-u_{h}\right\|_{L^{2}}$ & 1.099 & 1.709 & 1.952 & 1.876 & 1.988 & 2.019 \\
\cline { 2 - 7 } & $\left\|u-u_{h}\right\|_{H_{0}^{1}}$ & 0.580 & 0.838 & 1.018 & 0.926 & 0.989 & 1.093 \\
\hline \multirow{2}{*}{$v$} & $\left\|v-v_{h}\right\|_{L^{2}}$ & 1.156 & 1.500 & 1.924 & 1.476 & 1.457 & 2.072 \\
\cline { 2 - 7 } & $\left\|\partial_{z} v-\partial_{z} v_{h}\right\|_{L_{2}}$ & 0.770 & 0.894 & 1.032 & 0.941 & 1.004 & 1.081 \\
\hline \multirow{2}{*}{$p$} & $\left\|p-p_{h}\right\|_{L^{2}}$ & 0.479 & 0.790 & 1.022 & 0.905 & 0.977 & 1.110 \\
\hline
\end{tabular}

Table 1: Error orders for velocities and pressure with $\left(\mathcal{P}_{2}, \mathcal{P}_{1}\right)-\mathcal{P}_{0}$

following exact solution of the hydrostatic problem (1)-(3) in the unit squared:

$$
\begin{aligned}
& u(x, z)=\cos (2 \pi x) \sin (2 \pi z)-\sin (2 \pi z), \quad v(x, z)=-u(z, x), \\
& p(x, z)=2 \pi \cos (2 \pi x) .
\end{aligned}
$$

Note that condition $\int_{\Omega} p=0$ is satisfied and $\left.(u, v)\right|_{\partial \Omega}=0$.

Using $\left(\mathcal{P}_{2}, \mathcal{P}_{1}\right)-\mathcal{P}_{0}$, the approximated solution has been calculated and the absolute error for different norms has been computed, using different structured mesh sizes, obtaining the Figure 8 (in logarithmic scale). On the other hand, Table 1 shows concrete values representing the different error orders obtained, which are calculated from the operation $\log \left(e_{h_{2}} / e_{h_{1}}\right) / \log \left(h_{2} / h_{1}\right)$ when $h_{1}<h_{2}$ travel through the mesh sizes.

Both the slope of the segments in Figure 8 and the values in Table 1 suggest order $O(h)$ for $\left(u_{h}, v_{h}, p_{h}\right)$ in $H^{1} \times H_{z}^{1} \times L^{2}$, in agreement with Corollary 11. On the other hand, order $O\left(h^{2}\right)$ for velocity $\left(u_{h}, v_{h}\right)$ in $L^{2}$ is approximated numerically. Note that, though $u_{h}$ is taken in $\mathcal{P}_{2}$, optimal order for $u_{h}$ (order $O\left(h^{3}\right)$ in $L^{2}$ and $O\left(h^{2}\right)$ in $H^{1}$ ) is not obtained, due to the influence of the lower approximation of $v$ in $\mathcal{P}_{1}$.

These simulations, have been carried out in structured meshes, but the results in unstructured ones are similar. 


\subsection{About the stability of $\left(\mathcal{P}_{1, b}, \mathcal{P}_{1}\right)-\mathcal{P}_{1}$ and $\left(\mathcal{P}_{2}, \mathcal{P}_{1}\right)-\mathcal{P}_{1}$ for the hydro- static Stokes problem}

In this section we will use $\mathcal{P}_{1, b}$ finite elements to approximate horizontal velocity (choosing $U_{h}$ as in (48) and (52), respectively) and $\mathcal{P}_{1}$-continuous elements for the vertical velocity and for the pressure (choosing $V_{h}$ as in (54) and $P_{h}$ as in (50)). This choice denoted as $\left(\mathcal{P}_{1, b}, \mathcal{P}_{1}\right)-\mathcal{P}_{1}$ results from the elimination of some degrees of freedom from the "classical" mini-element $\mathcal{P}_{1, b}-\mathcal{P}_{1}$ (specifically, one bubble in each triangle for the vertical velocity). Similarly $\left(\mathcal{P}_{2}, \mathcal{P}_{1}\right)-\mathcal{P}_{1}$ results from the elimination of some degrees of freedom from the Taylor-Hood element $\mathcal{P}_{2}-\mathcal{P}_{1}$, for vertical velocity.

\section{The inf-sup condition $(I S)_{h}^{P}$}

It can be shown that $\left(\mathcal{P}_{1, b}, \mathcal{P}_{1}\right)-\mathcal{P}_{1}$ and $\left(\mathcal{P}_{2}, \mathcal{P}_{1}\right)-\mathcal{P}_{1}$ are Stokes-stable, in which we have relied so far for obtaining $(I S)_{h}^{P}$, for most usual unstructured meshes. Specifically, for those meshes that "are unstructured in the vertical direction" or, more precisely, mesh families that "do not tend to a vertical structure", as shown in [GR12] by using the macroelement technique [Ste90].

More in detail: we say that a macroelement is $x$-structured ( $y$-structured) if it can be split by an hyperplane orthogonal to the $O X$ axis ( $O Y$ axis), otherwise it is said $x^{-}$ unstructured ( $y$-unstructured). For instance, the mesh shown in figure 12a is $x$-structured and $y$-unstructured.

Under some geometrical assumptions for the mesh family $\mathcal{T}_{h}$, a sufficient condition for $\left(\mathcal{P}_{1, b}, \mathcal{P}_{1}\right)-\mathcal{P}_{1}$ to be Stokes-stable is [GR12]: $\mathcal{T}_{h}$ can be covered by a family of macroelements, $\mathcal{M}_{h}$, verifying the following conditions:

1. The macroelements in $\mathcal{M}_{h}$ contain one only interior element.

2. The macroelements in $\mathcal{M}_{h}$ are $y$-unstructured and furthermore they "do not tend to $y$-structured macroelements" when $h \rightarrow 0$.

Also $\left(\mathcal{P}_{2}, \mathcal{P}_{1}\right)-\mathcal{P}_{1}$ is Stokes-stable in this case, as long as another condition is also verified, which is related to the fact that some kind of "weak structure" is not presented in the mesh (and thus this condition is valid in common unstructured meshes). See [GR12] for more details.

In practice, in unstructured meshes built by usual mesh generators previous conditions can be assumed, as our numerical tests confirm for both $\left(\mathcal{P}_{1, b}, \mathcal{P}_{1}\right)-\mathcal{P}_{1}$ and $\left(\mathcal{P}_{2}, \mathcal{P}_{1}\right)-\mathcal{P}_{1}$ elements (see tests below).

\section{The inf-sup condition $(I S)_{h}^{V}$}

Up to our knowledge, there is not any theoretical result showing or denying condition $(I S)_{h}^{V}$ for $\left(\mathcal{P}_{1 b}, \mathcal{P}_{1}\right)-\mathcal{P}_{1}$ and $\left(\mathcal{P}_{2}, \mathcal{P}_{1}\right)-\mathcal{P}_{1}$. But, taking into account the following numerical tests, we can formulate some conjectures.

Indeed, according to Tests 6 and $7,(I S)_{h}^{V}$ could be satisfied in unstructured meshes but with $\gamma^{V}=\gamma^{V}(h)$. On the other hand, according to Test 8, we conjecture that $(I S)_{h}^{V}$ is satisfied in meshes which are $x$-structured but not $y$-structured, with $\gamma^{V}$ independent of $h$. 


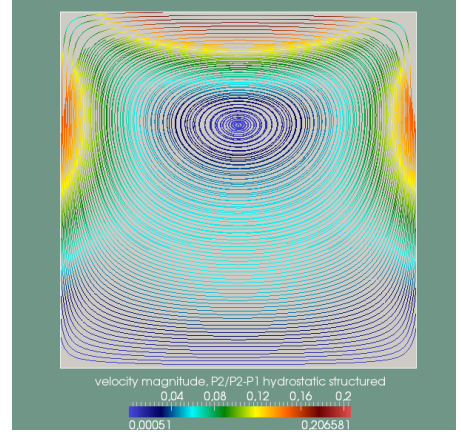

(a) Velocity streamlines

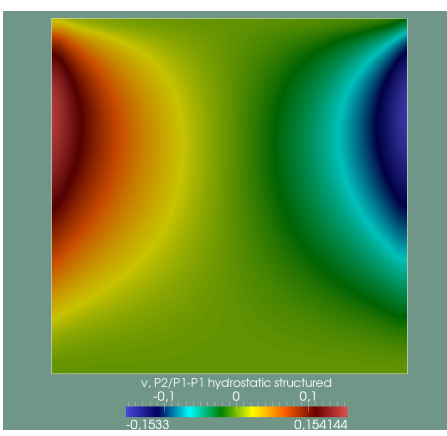

(b) Vertical velocity, $v_{h}$

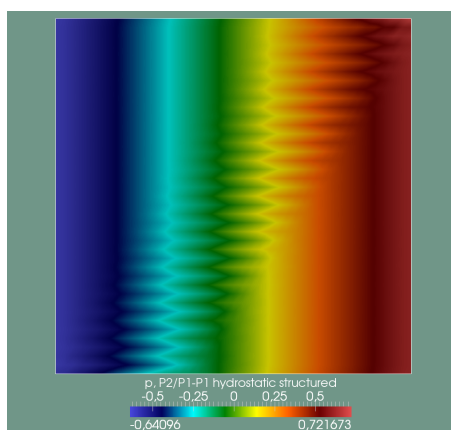

(c) Pressure, $p_{h}$

Figure 9: $\left(\mathcal{P}_{2}, \mathcal{P}_{1}\right)-\mathcal{P}_{1}$ approximation in a vertical structured mesh. Oscillations can be observed in pressure (but not in velocity).

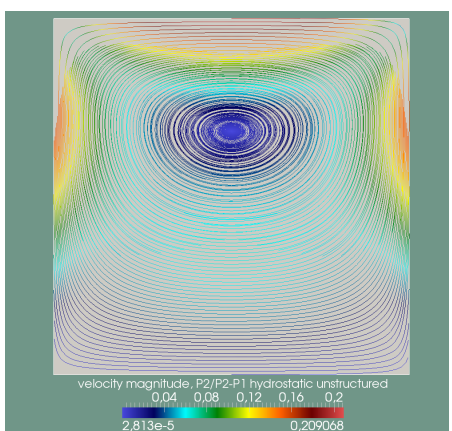

(a) Velocity streamlines

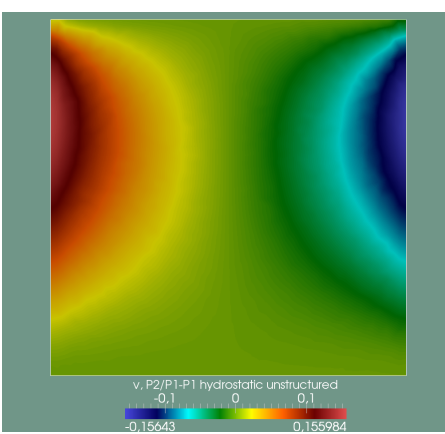

(b) Vertical velocity, $v_{h}$

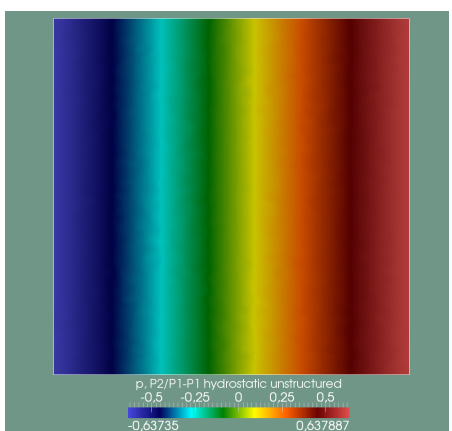

(c) Pressure, $p_{h}$

Figure 10: $\left(\mathcal{P}_{2}, \mathcal{P}_{1}\right)-\mathcal{P}_{1}$ approximation in an unstructured mesh. No oscillations in $v_{h}$ and $p_{h}$.

\section{Numerical tests}

Test $6\left(\left(\mathcal{P}_{1, b}, \mathcal{P}_{1}\right)-\mathcal{P}_{1}\right.$ and $\left(\mathcal{P}_{2}, \mathcal{P}_{1}\right)-\mathcal{P}_{1}$ cavity tests $)$. We consider the cavity test of Test 2 for either a structured $40 \times 40$ mesh or an unstructured one with $h \simeq 1 / 40$.

Both $\left(\mathcal{P}_{1 b}, \mathcal{P}_{1}\right)-\mathcal{P}_{1}$ and $\left(\mathcal{P}_{2}, \mathcal{P}_{1}\right)-\mathcal{P}_{1}$ have been used with similar results. For example, in $\left(\mathcal{P}_{2}, \mathcal{P}_{1}\right)-\mathcal{P}_{1}$ case, Figures 9 and 10 show that the qualitative behavior of horizontal and vertical velocity are correct both for structured and unstructured meshes. If the mesh is refined, similar pictures are obtained.

Also, the behavior of pressure is correct in the unstructured mesh (Figure 10c), but in the structured mesh the pressure present distinguishable oscillations (Figure 9c), according to the fact of $(I S)_{h}$ (and probably $(I S)_{h}^{P}$ ) is not verified in these types of meshes.

Test 7 (Error orders for $\left(\mathcal{P}_{1, b}, \mathcal{P}_{1}\right)-\mathcal{P}_{1}$ and $\left(\mathcal{P}_{2}, \mathcal{P}_{1}\right)-\mathcal{P}_{1}$ in unstructured meshes). $W e$ have repeated test 5 , in which an exact solution was provided.

The approximated solution and the absolute error for different norms have been calculated using unstructured meshes (as they are computed by the FreeFem++ environment), with mesh sizes in the range $h \simeq 2^{-2}, \ldots, 2^{-8}$. The results for $\left(\mathcal{P}_{1, b}, \mathcal{P}_{1}\right)-\mathcal{P}_{1}$ and 


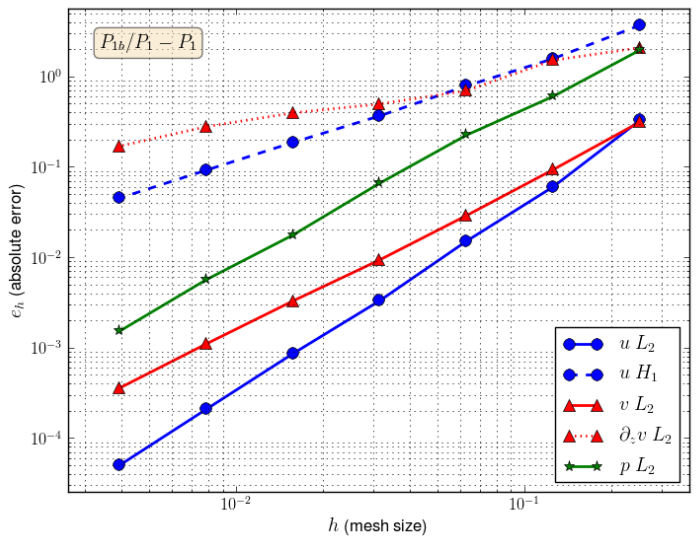

(a) Errors for $\left(\mathcal{P}_{1, b}, \mathcal{P}_{1}\right)-\mathcal{P}_{1}$

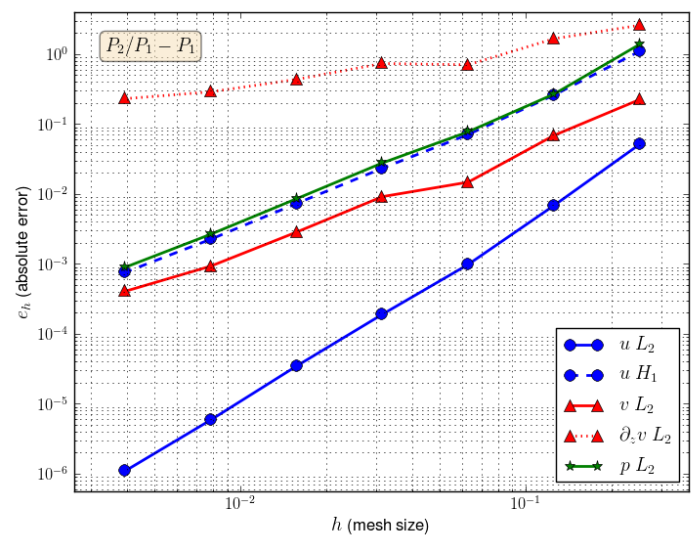

(b) Errors for $\left(\mathcal{P}_{2}, \mathcal{P}_{1}\right)-\mathcal{P}_{1}$

Figure 11: Velocity and pressure errors for $\left(\mathcal{P}_{1, b}, \mathcal{P}_{1}\right)-\mathcal{P}_{1}$ and $\left(\mathcal{P}_{2}, \mathcal{P}_{1}\right)-\mathcal{P}_{1}$ hydrostatic Stokes

\begin{tabular}{lrrrrrrr}
\hline$h_{\max }$ & & $2^{-3}$ & $2^{-4}$ & $2^{-5}$ & $2^{-6}$ & $2^{-7}$ & $2^{-8}$ \\
\hline \multirow{2}{*}{$u$} & $\left\|u-u_{h}\right\|_{L^{2}}$ & 2.442 & 1.998 & 2.187 & 1.945 & 2.044 & 2.059 \\
\cline { 2 - 7 } & $\left\|u-u_{h}\right\|_{H_{0}^{1}}$ & 1.229 & 1.001 & 1.102 & 0.976 & 1.020 & 1.027 \\
\hline \multirow{2}{*}{$v$} & $\left\|v-v_{h}\right\|_{L^{2}}$ & 1.752 & 1.688 & 1.629 & 1.510 & 1.578 & 1.623 \\
\hline \multirow{2}{*}{$p$} & $\left\|\partial_{z} v-\partial_{z} v_{h}\right\|_{L_{2}}$ & 0.463 & 1.117 & 0.490 & 0.327 & 0.508 & 0.715 \\
\hline
\end{tabular}

Table 2: Error orders for velocities and pressure $\left(\left(\mathcal{P}_{1, b}, \mathcal{P}_{1}\right)-\mathcal{P}_{1}\right.$ approximation $)$

$\left(\mathcal{P}_{2}, \mathcal{P}_{1}\right)-\mathcal{P}_{1}$ are shown in Figures 11 a and 11 , respectively. They allow us to deduce some conclusions, that are supported by the error orders given in Tables 2 and 3.

In the case of $\left(\mathcal{P}_{1, b}, \mathcal{P}_{1}\right)-\mathcal{P}_{1}$, optimal order for $u_{h}$ is obtained (order $O\left(h^{2}\right)$ in $L^{2}$ and $O(h)$ in $\left.H^{1}\right)$. In the case of $v_{h}$, optimal order is not clear, it seems that the orders obtained are slightly greater than $O\left(h^{1.5}\right)$ in $L^{2}$ and $O\left(h^{0.5}\right)$ in $H_{z}^{1}$. Also the order for $p_{h}$ in $L^{2}$ is in the interval $(3 / 2,2)$, confirming that, as expected, $(I S)_{h}^{P}$ holds for unstructured meshes.

On the other hand, for $\left(\mathcal{P}_{2}, \mathcal{P}_{1}\right)-\mathcal{P}_{1}$, orders for $u_{h}$ are around $O\left(h^{2.5}\right)$ in $L^{2}$ and $O\left(h^{1.5}\right)$ in $H^{1}$, then optimal orders $\left(O\left(h^{3}\right)\right.$ in $L^{2}$ and $O\left(h^{2}\right)$ in $\left.H^{1}\right)$ are not obtained in this case. The order for pressure seems, like in the previous case, to be greater than $O\left(h^{3 / 2}\right)$. But the behavior of $v_{h}$ is clearly worse than in the previous $\left(\mathcal{P}_{1, b}, \mathcal{P}_{1}\right)-\mathcal{P}_{1}$ case (around order $O(h)$ in $L^{2}$ and without order in $\left.H_{z}^{1}\right)$.

Test 8 (Error orders for $\left(\mathcal{P}_{1, b}, \mathcal{P}_{1}\right)-\mathcal{P}_{1}$ and $\left(\mathcal{P}_{2}, \mathcal{P}_{1}\right)-\mathcal{P}_{1}$ in a $x$-structured $/ y$-unstructured mesh). We have repeated Test 7 but now for the $x$-structured/y-unstructured given in Figure 12a. In these meshes, for $\left(\mathcal{P}_{1, b}, \mathcal{P}_{1}\right)-\mathcal{P}_{1}$, we can assure that $(I S)_{h}^{P}$ is verified (in usual unstructured meshes, it was enounced above), but we do not know whether $(I S)_{h}^{V}$ holds or not. The results for $\left(\mathcal{P}_{1, b}, \mathcal{P}_{1}\right)-\mathcal{P}_{1}$ (see Figure $12 b$ and Table 5) suggest optimal order $O(h)$ for $v_{h}$ in $H_{z}^{1}(\Omega)$ and then allow us to conjecture that $(I S)_{h}^{V}$ holds. 


\begin{tabular}{lrrrrrrr}
\hline$h_{\max }$ & & $2^{-3}$ & $2^{-4}$ & $2^{-5}$ & $2^{-6}$ & $2^{-7}$ & $2^{-8}$ \\
\hline \multirow{2}{*}{$u$} & $\left\|u-u_{h}\right\|_{L^{2}}$ & 2.912 & 2.803 & 2.369 & 2.458 & 2.561 & 2.429 \\
\cline { 2 - 7 } & $\left\|u-u_{h}\right\|_{H_{0}^{1}}$ & 2.086 & 1.872 & 1.605 & 1.676 & 1.697 & 1.576 \\
\hline \multirow{2}{*}{$v$} & $\left\|v-v_{h}\right\|_{L^{2}}$ & 1.706 & 2.222 & 0.687 & 1.686 & 1.617 & 1.198 \\
\hline \multirow{2}{*}{$p$} & $\left\|\partial_{z} v-\partial_{z} v_{h}\right\|_{L_{2}}$ & 0.641 & 1.240 & -0.062 & 0.754 & 0.593 & 0.326 \\
\hline
\end{tabular}

Table 3: Error orders for velocities and pressure $\left(\left(\mathcal{P}_{2}, \mathcal{P}_{1}\right)-\mathcal{P}_{1}\right.$ approximation $)$

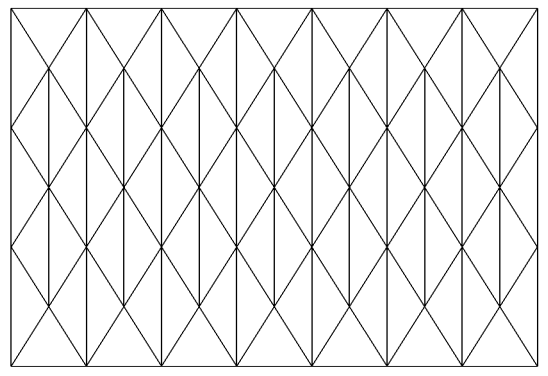

(a) Mesh type used for the experiment

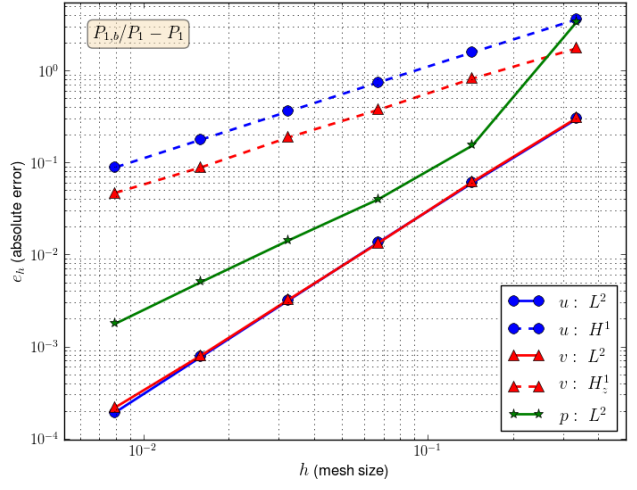

(b) Errors for $\left(\mathcal{P}_{1, b}, \mathcal{P}_{1}\right)-\mathcal{P}_{1}$

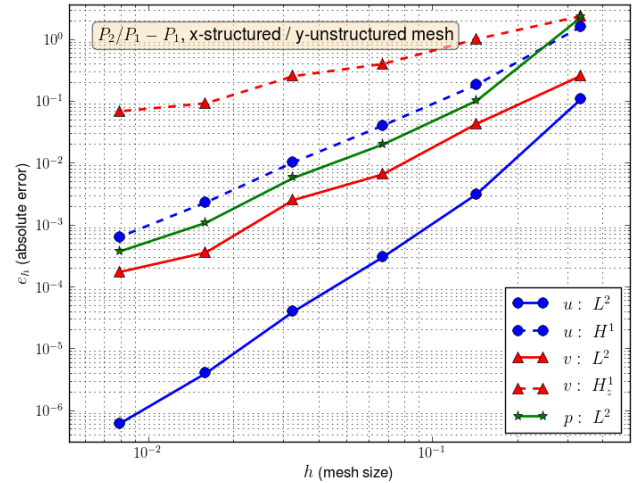

(c) Errors for $\left(\mathcal{P}_{2}, \mathcal{P}_{1}\right)-\mathcal{P}_{1}$

Figure 12: Error orders in a $x$-structured/ $y$-unstructured mesh.

\begin{tabular}{lrrrrrr}
\hline$h_{\max }$ & $2^{-3}$ & $2^{-4}$ & $2^{-5}$ & $2^{-6}$ & $2^{-7}$ \\
\hline \multirow{2}{*}{$u$} & $\left\|u-u_{h}\right\|_{L^{2}}$ & 1.894 & 1.961 & 1.990 & 1.997 & 1.999 \\
\cline { 2 - 7 } & $\left\|u-u_{h}\right\|_{H_{0}^{1}}$ & 0.977 & 0.986 & 0.997 & 1.000 & 1.000 \\
\hline \multirow{2}{*}{$v$} & $\left\|v-v_{h}\right\|_{L^{2}}$ & 1.893 & 2.009 & 1.950 & 1.973 & 1.870 \\
\hline \multirow{2}{*}{$p$} & $\left\|\partial_{z} v-\partial_{z} v_{h}\right\|_{L_{2}}$ & 0.896 & 1.027 & 0.948 & 1.059 & 0.920 \\
\hline
\end{tabular}

Table 4: Error orders for $\left(\mathcal{P}_{1, b}, \mathcal{P}_{1}\right)-\mathcal{P}_{1}$ in a $x$-structured $/ y$-unstructured mesh 


\begin{tabular}{lrrrrrr}
\hline$h_{\max }$ & & $2^{-3}$ & $2^{-4}$ & $2^{-5}$ & $2^{-6}$ & $2^{-7}$ \\
\hline \multirow{2}{*}{$u$} & $\left\|u-u_{h}\right\|_{L^{2}}$ & 4.177 & 3.082 & 2.791 & 3.230 & 2.681 \\
\cline { 2 - 7 } & $\left\|u-u_{h}\right\|_{H_{0}^{1}}$ & 2.559 & 1.992 & 1.883 & 2.105 & 1.834 \\
\hline \multirow{2}{*}{$v$} & $\left\|v-v_{h}\right\|_{L^{2}}$ & 2.112 & 2.471 & 1.318 & 2.750 & 1.040 \\
\hline \multirow{2}{*}{$p$} & $\left\|\partial_{z} v-\partial_{z} v_{h}\right\|_{L_{2}}$ & 1.010 & 1.217 & 0.624 & 1.427 & 0.425 \\
\hline
\end{tabular}

Table 5: Error orders for $\left(\mathcal{P}_{2}, \mathcal{P}_{1}\right)-\mathcal{P}_{1}$ in a $x$-structured $/ y$-unstructured mesh

On the other hand, we cannot assure $(I S)_{h}^{P}$ for $\left(\mathcal{P}_{2}, \mathcal{P}_{1}\right)-\mathcal{P}_{1}$ in this particular mesh (because this mesh verifies a "weak structure" condition, see [GR12] for more details). Thus we cannot assure the stability of $\left(\mathcal{P}_{2}, \mathcal{P}_{1}\right)-\mathcal{P}_{1}$.

Anyway, our numerical tests suggest stability of $\left(\mathcal{P}_{2}, \mathcal{P}_{1}\right)-\mathcal{P}_{1}$ in this kind of meshes, in the sense of $(I S)_{h}^{P}$. Specifically, orders for $\left(\mathcal{P}_{2}, \mathcal{P}_{1}\right)-\mathcal{P}_{1}$ are comparable with the ones obtained in unstructured meshes (Test 7), and even better for $u_{h}$ and $p_{h}$ : near to optimal order for $u_{h}$ (near to $O\left(h^{3}\right)$ in $L^{2}$ and $O\left(h^{2}\right)$ in $H^{1}$ ) and greater than $O\left(h^{3 / 2}\right)$ for pressure. No kind of spurious oscillations were observed in the contour plots of $p_{h}$. Note that orders for $v_{h}$ are worse than in the $\left(\mathcal{P}_{1, b}, \mathcal{P}_{1}\right)-\mathcal{P}_{1}$ case: around order $O(h)$ in $L^{2}$ and $O\left(h^{1 / 2}\right)$ in $H_{z}^{1}$. This fact could be related to $(I S)_{h}^{V}$.

Then, this would be an example of local unstability (in a family of macroelements) does not imply global unstability, in the sense of $(I S)_{h}^{P}$.

\section{Numerical simulations with different domains}

We present some numerical tests with non-constant bottom, with the aim of showing, in practice, some of the advantages of the hydrostatic Stokes scheme (37). Specifically, we exploit its flexibility for choosing different domains, which are approximated by standard finite element meshes (not necessary structured). Convenient techniques, such as mesh adaptivity, are applied without additional difficulties.

Test 9. We consider a non convex $2 D$ domain defined by the surface interval $S=[0,1]$ and a depth function $D(x)$, whose minimum value is reached at $x=1 / 2$ and the maximum at $x=0$ and $x=1$. We set $\mathbf{f}=0$ and fix the usual boundary conditions (8)-(9), where the wind traction has a constant value $\mathbf{g}_{s}=0.5$ on $\Gamma_{S}$ (which, keeping in mind the adherence condition of $u$ on $\Gamma_{l}$ will produce boundary layers at the corners $x=0, z=0$ and $x=1, z=0$ ).

We consider an unstructured mesh of $\Omega$ with 31 nodes on each boundary. A standard finite elements software package (FreeFem ++ , [PHLHM]) has been used for mesh construction. Moreover, the facilities of FreeFem ++ for mesh adaptivity has been exploited and refined meshes have been obtained, depending on the indicator function

$$
u_{h} /\left\|u_{h}\right\|_{\infty}+v_{h} /\left\|v_{h}\right\|_{\infty}+p_{h} /\left\|p_{h}\right\|_{\infty} .
$$

Figures $13 a$ and $13 b$ show the meshes obtained after four solving plus mesh adaptation steps, using $\left(\mathcal{P}_{2}, \mathcal{P}_{1}\right)-\mathcal{P}_{0}$ and $\left(\mathcal{P}_{2}, \mathcal{P}_{1}\right)-\mathcal{P}_{1}$, respectively. Figures $13 c-13 f$ show the velocity 


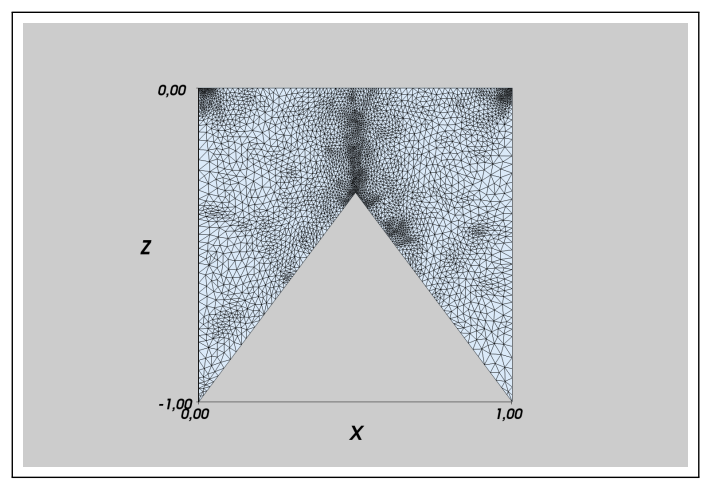

(a) $\left(\mathcal{P}_{2}, \mathcal{P}_{1}\right)-\mathcal{P}_{0}$ refined mesh

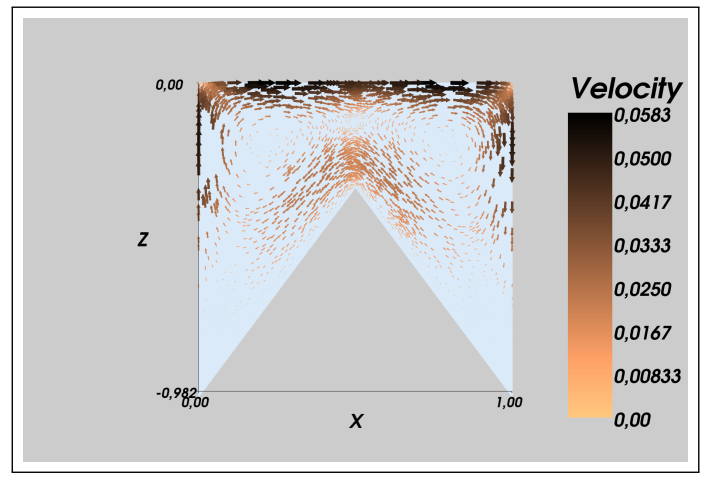

(c) $\left(\mathcal{P}_{2}, \mathcal{P}_{1}\right)-\mathcal{P}_{0}$ velocity field

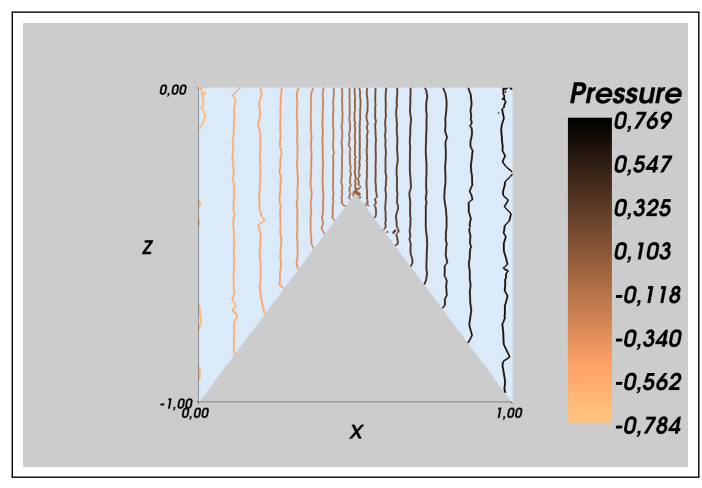

(e) $\left(\mathcal{P}_{2}, \mathcal{P}_{1}\right)-\mathcal{P}_{0}$ pressure isolines

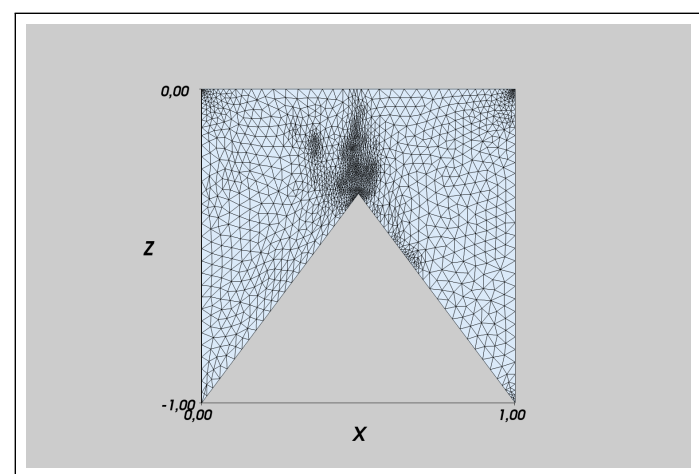

(b) $\left(\mathcal{P}_{2}, \mathcal{P}_{1}\right)-\mathcal{P}_{1}$ refined mesh

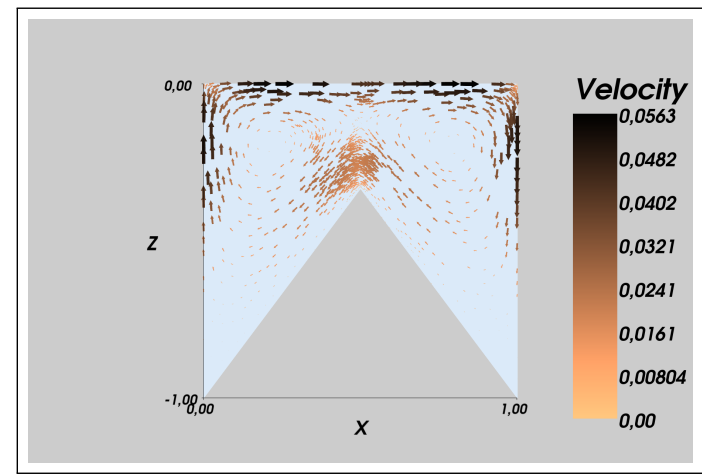

(d) $\left(\mathcal{P}_{2}, \mathcal{P}_{1}\right)-\mathcal{P}_{1}$ velocity field

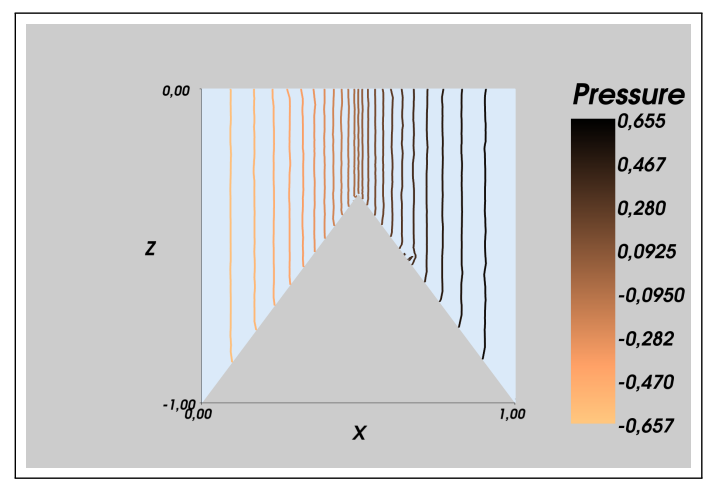

(f) $\left(\mathcal{P}_{2}, \mathcal{P}_{1}\right)-\mathcal{P}_{1}$ pressure isolines

Figure 13: Numerical tests in a non convex domain: $\left(\mathcal{P}_{2}, \mathcal{P}_{1}\right)-\mathcal{P}_{0}$ (left column) and $\left(\mathcal{P}_{2}, \mathcal{P}_{1}\right)-\mathcal{P}_{1}$ (right column) 


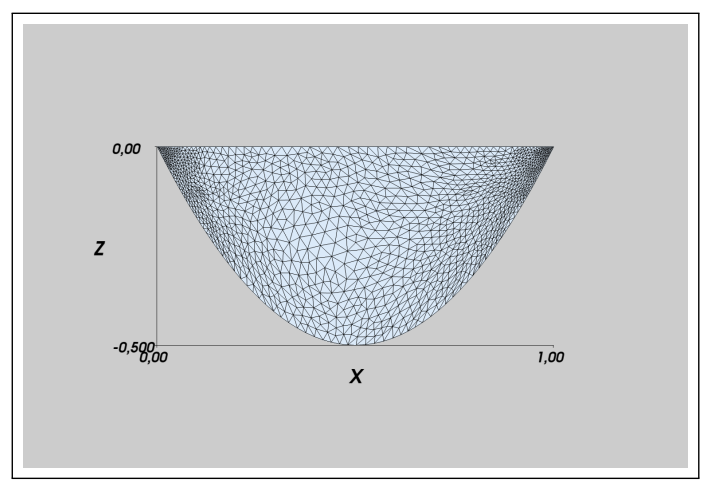

(a) $\left(\mathcal{P}_{2}, \mathcal{P}_{1}\right)-\mathcal{P}_{0}$ refined mesh

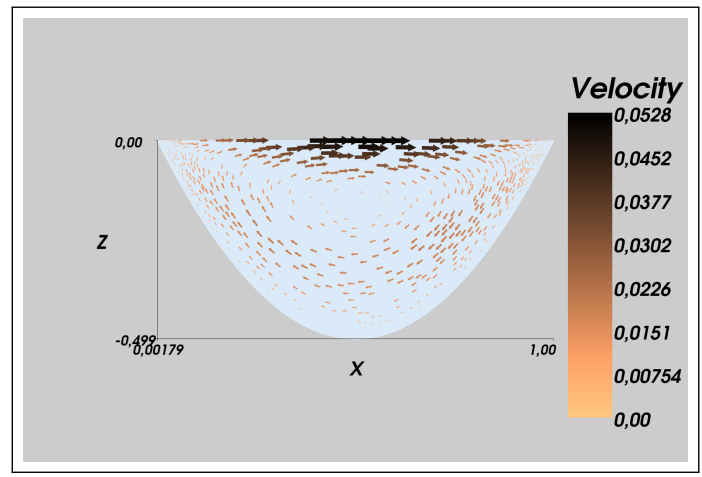

(c) $\left(\mathcal{P}_{2}, \mathcal{P}_{1}\right)-\mathcal{P}_{0}$ velocity field

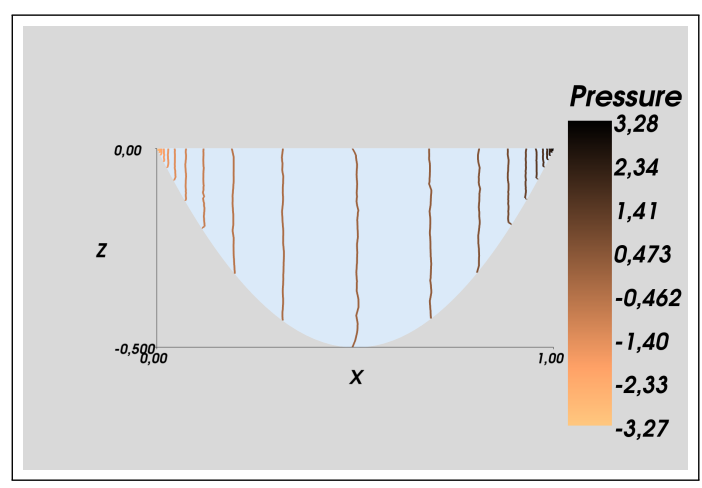

(e) $\left(\mathcal{P}_{2}, \mathcal{P}_{1}\right)-\mathcal{P}_{0}$ pressure isolines

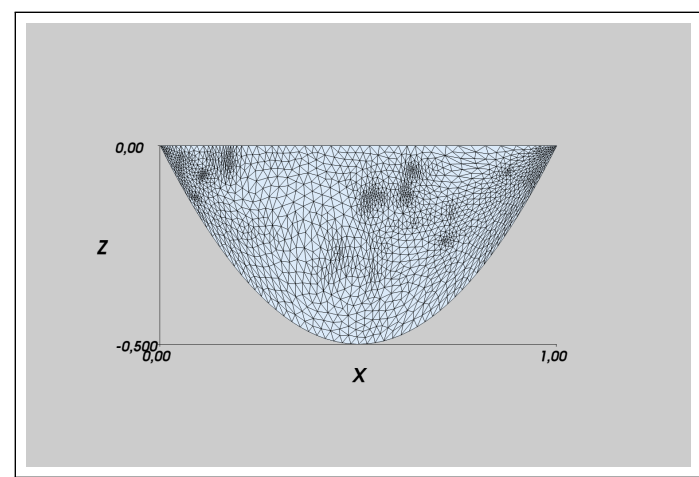

(b) $\left(\mathcal{P}_{2}, \mathcal{P}_{1}\right)-\mathcal{P}_{1}$ refined mesh

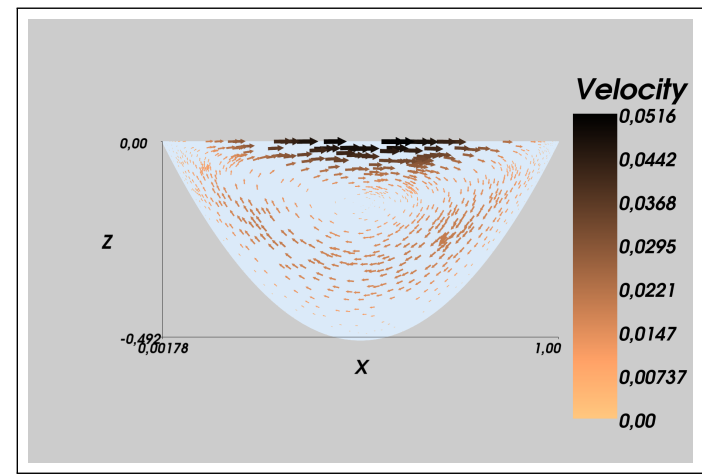

(d) $\left(\mathcal{P}_{2}, \mathcal{P}_{1}\right)-\mathcal{P}_{1}$ velocity field

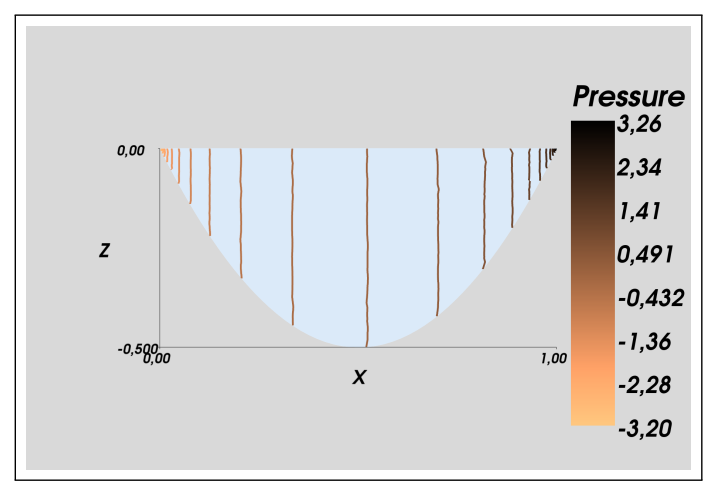

(f) $\left(\mathcal{P}_{2}, \mathcal{P}_{1}\right)-\mathcal{P}_{1}$ pressure isolines

Figure 14: Numerical tests in a convex domain without sidewall talus: $\left(\mathcal{P}_{2}, \mathcal{P}_{1}\right)-\mathcal{P}_{0}$ (left column) and $\left(\mathcal{P}_{2}, \mathcal{P}_{1}\right)-\mathcal{P}_{1}$ (right column). 
field and pressure isolines. As we expected, both of them are, qualitatively, suitable results. Moreover, they agree with similar tests given in literature, where reduced integro-differential formulations (in structured meshes) are employed (see e.g. [CGS12], Test 3). Specifically, two large vortices, one on each side of the interior mountain, are obtained, while pressure is constant in depth, increasing horizontally (faster at the mountain peak of the bottom).

Note that pressure graphic for $\left(\mathcal{P}_{2}, \mathcal{P}_{1}\right)-\mathcal{P}_{1}$ is better (presents less oscillations) than for $\left(\mathcal{P}_{2}, \mathcal{P}_{1}\right)-\mathcal{P}_{0}$, needing the latter one a higher mesh refinement (hence a higher computational effort). As counterpart, the velocity seems better for $\left(\mathcal{P}_{2}, \mathcal{P}_{1}\right)-\mathcal{P}_{0}$. Note that, as predicted in Section 6.4, the random unstructured meshes obtained by mesh refinement provide stability for $\left(\mathcal{P}_{2}, \mathcal{P}_{1}\right)-\mathcal{P}_{1}$.

Test 10. We only change the depth function $D(x)$ in $S=[0,1]$ being now $D=0$ at $x=0$ and $x=1$ (hence, there are not sidewalls and $\Gamma_{l}=\emptyset$ ) and reaches its maximum at $x=0.5$ with $D(0.5)=0.5$. Figures $14 a$ and $14 b$ show the refined mesh obtained after four solving +adaptation steps (using the same indicator (57)), starting from a triangulation with 31 nodes on $\Gamma_{S}$ and $\Gamma_{b}$.

Note that, unlike what happens for schemes derived from the reduced formulation (14)(16) [CG00, CR, CR05, GR05, CGS12], now it is not necessary to impose a talus $(D(x) \geq$ $\tau>0)$ in the domain. In fact, as reflected by figures $14 c-14 f$, both $\left(\mathcal{P}_{2}, \mathcal{P}_{1}\right)-\mathcal{P}_{0}$ and $\left(\mathcal{P}_{2}, \mathcal{P}_{1}\right)-\mathcal{P}_{1}$ provide correct approximations. Observe that one only vortex is obtained and extreme values of pressure are concentrated near the "coast", where depth vanishes.

Note also that it is not usual to find these kinds of experiments in literature, due to the difficulties presented by reduced integro-differential formulations when the domain has no talus.

\section{References}

[AG01] P. Azérad and F. Guillén. Mathematical justification of the hydrostatic approximation in the primitive equations of geophysical fluid dynamics. Siam J. Math. Ana., 33(4):847-859, 2001.

[Azé94] P. Azérad. Analyse et approximation du problème de Stokes dans un bassin peu profond. C. R. Acad. Sci. Paris Sér. I Math., 318(1):53-58, 1994.

[Azé96] P. Azérad. Analyse des quations de Navier-Stokes en bassin peu profond et de l'quation de transport. PhD thesis, Neuchtel, 1996.

[BF91] F. Brezzi and M. Fortin. Mixed and Hybrid Finite Element Methods. SpringerVerlag, New-York, 1991.

[BL92] O. Besson and M.R. Laydi. Some estimates for the anisotropic Navier-Stokes equations and for the hydrostatic approximation. Math. Mod. and Num. Anal, Vol. 26(7):855-865, 1992.

[CB09] B. Cushman-Roisin and J. M. Beckers. Introduction to Geophysical Fluid Dynamics - Physical and Numerical Aspects. Academic Press, 2009. 
[CG00] T. Chacón-Rebollo and F. Guillén-González. An intrinsic analysis of the hydrostatic approximation of Navier-Stokes equations. C. R. Acad. Sci. Paris, Srie I(330):841-846, 2000 .

[CGS12] T. Chacón-Rebollo, M. Gómez-Mármol, and I. Sánchez-Muñoz. Numerical solution of the primitive equations of the ocean by the orthogonal sub-scales vms method. Applied Numerical Mathematics, 62:342359, 2012.

[Cia78] P.G. Ciarlet. The Finite Element Method for Elliptic Problems. North-Holland, Amsterdam, 1978.

[CR $\quad$ T. Chacón-Rebollo and D. Rodríguez-Gómez. Prismatic finite element solution of the primitive equations of the ocean. .

[CR05] T. Chacón-Rebollo and D. Rodríguez-Gómez. A numerical solver for the primitive equations of the ocean using term-by-term stabilization. Appl. Numer. Math., 55(1):1-31, 2005.

[CT07] C. Cao and E.S. Titi. Global well-posedness of the three-dimensional viscous primitive equations of large scale ocean and atmosphere dynamics. Annals of Mathematics, 166:245-267, 2007.

[EG04] A. Ern and J.-L. Guermond. Theory and Practice of Finite Elements. Springer, 2004 .

[GMR01] F. Guillén-González, N. Masmoudi, and M.A. Rodríguez-Bellido. Anisotropic estimates and strong solutions of the primitive equations. Differential and Integral Equations, 14(11):1381-1408, 2001.

[GR05] F. Guillén-González and D. Rodríguez-Gómez. Bubble finite elements for the primitive equations of the ocean. Numerische Mathematik, 101:689-728, 2005.

[GR12] F. Guillén-González and J.R. Rodríguez-Galván. On the stability of approximations for the stokes problem using different finite element spaces for each component of the velocity. Submitted. Available at http://www.uca.es/dpto/C101/pags-personales/rafael.rodriguez/ papers/stokes-velocity-fespaces.pdf, 2012.

[Kim13] M. Kimmritz. Equal-order Finite Elements of Hydrostatic Flow Problems. PhD thesis, Kiel, 2013.

[LTW92a] J.-L. Lions, R. Temam, and S. Wang. New formulations of the primitive equations of the atmosphere and applications. Nonlinearity, 5:237-288, 1992.

[LTW92b] J.-L. Lions, R. Temam, and S. Wang. On the equations of large scale ocean. Nonlinearity, 5:1007-1053, 1992.

[Ort04] F. Ortegón-Gallego. On distributions independent of $\mathrm{xn}$ in certain noncylindrical domains and a de rham lemma with a non-local constraint. Nonlinear Analysis: Theory, Methods $\&$ Applications, 59(3):335 - 345, 2004. 
[Ped87] J. Pedlosky. Geophysical Fluid Dynamics. Springer-Verlag, New York, USA, 1987.

[PHLHM] O. Pironneau, F. Hecht, A. Le Hyaric, and J. Morice. FreeFEM++, http: //www.freefem.org/.

[Ste90] R. Stenberg. A technique for analysing finite elements methods for viscuous incompressible flow. International Journal for Numerical Methods in Fluids, 11:835-948, 1990.

[Tem77] R. Temam. Navier-Stokes equations: theory and numerical analysis. Amer. Mathematical Society, 1977.

[Tem03] R. Temam. Some mathematical aspects of geophysical fluid dynamic equations. Milan J. Math., 71:175-198, 2003.

[TZ04] R. Temam and M. Ziane. Some mathematical problems in geophysical fluid dynamics. In Handbook of Mathematical Fluid Dynamics, volume 3, pages 535-658. Friedlander and D. Serre Editors, Elsevier, 2004.

[Zia95] M. Ziane. Regularity results for stokes type systems. Applicable Analysis, 58:263-292, 1995. 